\title{
Sensitivity of the Brewer-Dobson Circulation and Polar Vortex Variability to Parameterized Nonorographic Gravity Wave Drag in a High-Resolution Atmospheric Model
}

\author{
I. POLICHTCHOUK \\ Department of Meteorology, University of Reading, and European Centre for Medium-Range \\ Weather Forecasts, Reading, United Kingdom \\ T. G. SHEPHERD \\ Department of Meteorology, University of Reading, Reading, United Kingdom \\ R. J. HOGAN \\ European Centre for Medium-Range Weather Forecasts, and Department of Meteorology, \\ University of Reading, Reading, United Kingdom \\ P. BECHTOLD \\ European Centre for Medium-Range Weather Forecasts, Reading, United Kingdom
}

(Manuscript received 9 October 2017, in final form 6 February 2018)

\begin{abstract}
The role of parameterized nonorographic gravity wave drag (NOGWD) and its seasonal interaction with the resolved wave drag in the stratosphere has been extensively studied in low-resolution (coarser than $1.9^{\circ} \times$ $2.5^{\circ}$ ) climate models but is comparatively unexplored in higher-resolution models. Using the European Centre for Medium-Range Weather Forecasts Integrated Forecast System at $0.7^{\circ} \times 0.7^{\circ}$ resolution, the wave drivers of the Brewer-Dobson circulation are diagnosed and the circulation sensitivity to the NOGW launch flux is explored. NOGWs are found to account for nearly $20 \%$ of the lower-stratospheric Southern Hemisphere (SH) polar cap downwelling and for less than $10 \%$ of the lower-stratospheric tropical upwelling and Northern Hemisphere (NH) polar cap downwelling. Despite these relatively small numbers, there are complex interactions between NOGWD and resolved wave drag, in both polar regions. Seasonal cycle analysis reveals a temporal offset in the resolved and parameterized wave interaction: the NOGWD response to altered source fluxes is largest in midwinter, while the resolved wave response is largest in the late winter and spring. This temporal offset is especially prominent in the SH. The impact of NOGWD on sudden stratospheric warming (SSW) life cycles and the final warming date in the $\mathrm{SH}$ is also investigated. An increase in NOGWD leads to an increase in SSW frequency, reduction in amplitude and persistence, and an earlier recovery of the stratopause following an SSW event. The SH final warming date is also brought forward when NOGWD is increased. Thus, NOGWD is still found to be a very important parameterization for stratospheric dynamics even in a high-resolution atmospheric model.
\end{abstract}

\section{Introduction}

The wave-driven stratospheric overturning circulation, with air rising and dynamically cooling in the tropics and descending and dynamically warming in the extratropics, exerts a crucial control on stratospheric

Corresponding author: I. Polichtchouk, i.polichtchouk@reading. ac.uk temperature and thereby on winds (e.g., Shepherd 2000). It also plays a key role in the transport of water vapor, ozone, and other chemical species. This mass transport circulation is named the Brewer-Dobson circulation (BDC). Faithfully representing the BDC in numerical weather and climate prediction models is vital for accurate stratospheric temperature distribution and chemistry. Accurate representation of stratospheric circulation, in turn, is important for tropospheric predictability on 
medium-range and seasonal time scales (e.g., Baldwin and Dunkerton 2001; Douville 2009; Sigmond et al. 2013), as well as for getting the correct background information into the data assimilation system, given the deep weighting functions of the operational nadir temperature sounders (e.g., Polavarapu et al. 2005).

Rossby and gravity wave breaking and saturation in the middle atmosphere drive the BDC [for a review on the BDC see Butchart (2014)]. In most models, small-scale orographic and nonorographic gravity wave breaking and saturation is parameterized [for a review on gravity waves and their parameterization in models see Fritts and Alexander (2003) and Plougonven and Zhang (2014)]. Throughout this paper we will refer to parameterized nonorographic gravity wave drag (NOGWD) and parameterized orographic gravity wave drag (OGWD). OGWD is an important source of stratospheric drag in both hemispheres in low-resolution models (e.g., McLandress and Shepherd 2009a; McLandress et al. 2012), with NOGWD playing a lesser role. However, the role of parameterized wave drag should diminish at higher resolution when the wave drag is increasingly resolved by the model. Therefore, the first aim of this study is to diagnose the role of the parameterized waves in driving the tropical upwelling and polar cap downwelling at relatively high horizontal resolution using the European Centre for Medium-Range Weather Forecasts (ECMWF) Integrated Forecast System (IFS). The downward-control principle of Haynes et al. (1991), which expresses the BDC as a response to breaking and saturating waves aloft, is used to separate the drivers of the BDC into OGWD, NOGWD, and resolved wave drag. Thus far, such a separation has only been carried out for low horizontal (coarser than $1.9^{\circ} \times 2.5^{\circ}$ ) and vertical (coarser than $1 \mathrm{~km}$ in the lower stratosphere) resolution stratosphere resolving climate models.

Diagnostically, OGWD is found to be a minor contributor to drag in the IFS at TL255L137 resolution ( $80 \mathrm{~km}$ in the horizontal and $\sim 300 \mathrm{~m}$ in the vertical in the lower stratosphere) whereas NOGWD remains important, especially in the SH. Therefore, the second aim of this study is to assess the impact of NOGWD flux perturbations on the strength of the BDC, and on the resolved wave drag over the seasonal cycle. The seasonal cycle has received relatively little attention in the studies of parameterized and resolved wave drag interaction (e.g., Cohen et al. 2013, 2014; Sigmond and Shepherd 2014), which have focused on the time-mean response. In the SH stratosphere, the resolved and parameterized wave drag exhibit distinct seasonality: the resolved wave drag maximizes in late winter/spring (Randel 1988; Quintanar and Mechoso 1995) and the parameterized wave drag in midwinter (Pulido and Thuburn 2008). Shaw et al. (2009) studied the interaction between reduced parameterized gravity wave drag (GWD) (via lowering the upper boundary condition) and resolved drag in the context of the seasonal cycle in polar regions in the Canadian Middle Atmosphere Model (CMAM) at low resolution. The study found that reducing parameterized GWD altered resolved wave drag leading to polar cap upper-stratospheric downwelling changing to upwelling in the $\mathrm{NH}$, and to a shift of maximum downwelling from November to December in the SH. The final aim of this study is to develop those concepts further with a high-resolution model and in the context of NOGWD perturbations. For example, the dominant $\mathrm{NH}$ drag in CMAM was OGWD, and this will have a very different response to wind changes than NOGWD, which has a broad phase speed spectrum.

Climatologically, NOGWD perturbations have a relatively small effect on the NH BDC in the IFS. However, NOGWD has a significant impact on the temporal evolution of polar dynamics, which is investigated here in the context of $\mathrm{NH}$ sudden stratospheric warming (SSW) life cycles and in particular Arctic polar night jet oscillation (PJO) events, which are long lived and have a stronger influence on the troposphere than other SSWs (Hitchcock et al. 2013; Hitchcock and Shepherd 2013; Hitchcock and Simpson 2014).

The paper is organized as follows. Section 2 briefly reviews the model, the experimental setup, and the diagnostics used. Section 3 reviews the middle atmosphere momentum budget in the control run. In section 4 , the BDC - split into its different wave drivers-is diagnosed for the control run with the free-running model. The impact of NOGWD flux on the BDC climatology and seasonal cycle is also discussed in this section. In section 5, the impact of NOGWD on the SSWs in the NH is discussed. Finally, a summary and conclusions are given in section 6.

\section{Methods}

\section{a. Model description and setup}

The IFS is a global semi-Lagrangian pseudospectral model developed and used for operational forecasts. The detailed description of its dynamical core and the physical parameterizations - as used in cycle 43R1 - can be found in ECMWF (2016). Here, IFS is run at TL255 spectral truncation with a linear Gaussian grid (grid spacing of $\sim 80 \mathrm{~km}$ ) and a time-step size of $1800 \mathrm{~s}$. The vertical domain is discretized into 137 levels (the resolution is $\sim 300 \mathrm{~m}$ at $100 \mathrm{hPa}$, coarsening to $\sim 1.5 \mathrm{~km}$ at $1 \mathrm{hPa}$ ) and the model top is located at $0.01 \mathrm{hPa}$. To prevent wave reflection at the model top, a fourth-order 
hyper-diffusion $\left(\nabla^{4}\right)$ is applied on vorticity, divergence, and temperature fields above $10 \mathrm{hPa}$ to damp vertically propagating waves. The hyper-diffusion $e$-folding time scale on the largest resolved wavenumber decreases from $0.65 \mathrm{~h}$ at $10 \mathrm{hPa}$ to $0.03 \mathrm{~h}$ at the model top. In addition, a first-order diffusion $(\nabla)$ is applied on the divergence field only above $1 \mathrm{hPa}$. The diffusion $e$-folding time scale on the largest resolved wavenumber decreases from $0.1 \mathrm{~h}$ at $1 \mathrm{hPa}$ to $0.02 \mathrm{~h}$ at the model top. Both "sponges" damp the zonal-mean fields (i.e., apply diffusion on the zonal wavenumber $m=0$ coefficients).

The nonorographic gravity wave drag parameterization in the IFS follows Scinocca (2003). Orr et al. (2010) discuss in detail the specific implementation and beneficial effect of this parameterization on the middle atmosphere circulation in the IFS. In the default setting, the momentum source is represented by a broad spectrum of wave speeds (half-width of $150 \mathrm{~m} \mathrm{~s}^{-1}$ ) discretized into 25 variable-resolution phase-speed bins and launched at $450 \mathrm{hPa}$. The 450-hPa launch level implies that NOGWs can break in the upper troposphere and lower stratosphere on encountering critical levels, such as when the subtropical jets terminate in the lower stratosphere. The orographic gravity wave drag parameterization in the IFS follows Lott and Miller (1997).

Two different experimental protocols are followed: 1) an ensemble of 4-yr forecasts; and 2) nudged 7-month forecasts, where the troposphere below $500 \mathrm{hPa}$ is nudged toward ERA-Interim reanalyses (Dee et al. 2011) to constrain planetary and synoptic-wave forcing from the troposphere. The "free running" setup 1 allows us to answer the question of how the model statistics respond to NOGWD changes. Setup 2 allows us to study the response of internal middle atmosphere dynamics to changes in NOGWD, specifically to reproduce the evolution of the 2006 PJO life cycle. All simulations are forced by prescribed daily varying observed sea surface temperatures.

In the free-running setup, eight 4-yr forecasts are initialized 1 year apart with the first forecast starting on 1 August 2004. The first month is disregarded as a spinup period. This procedure samples years from 2004 to 2015 and generates 32 (nonindependent) years of data. Three simulations are performed: one with the default NOGWD launch spectrum amplitude of $3.75 \mathrm{mPa}$, one with the NOGWD launch spectrum amplitude reduced to $1 \mathrm{mPa}$, and one with the NOGWD launch spectrum amplitude increased to $14 \mathrm{mPa}$. The study of such a broad range of flux amplitudes is motivated by Scheffler and Pulido (2017) who find, using a data assimilation technique, that the optimal launch momentum flux in the SH lower stratosphere can fluctuate between 4 and 0.25 times the reference value over the seasonal cycle. In all cases the amplitude of the launch spectrum is reduced in amplitude by $75 \%$ in the tropics. ${ }^{1}$

In the nudged setup, relative vorticity and temperature fields are relaxed via Newtonian relaxation to the ERA-Interim reanalysis fields on the terrainfollowing model levels below $500 \mathrm{hPa}$. The fields only up to total wavenumber 61 in the spherical harmonic expansion are nudged. The relaxation time scale is $12 \mathrm{~h}$ for relative vorticity and 5 days for temperature. To study the 2006 PJO life cycle, forecasts with five ensemble members each are started on 1 November 2005. As in the free-running setup, three forecasts with different NOGWD launch spectrum amplitudes are performed.

Henceforth, all forecasts using the default NOGWD launch amplitude will be referred to as the "control run," the reduced NOGWD launch amplitude forecasts will be referred to as the "reduced NOGWD run," and the increased NOGWD launch amplitude forecasts will be referred to as the "increased NOGWD run."

Fields are output every $6 \mathrm{~h}$ to sample the diurnal cycle. As noted in Seviour et al. (2012) and Sakazaki et al. (2015) there is a strong diurnal cycle in the zonal-mean fields in the stratosphere-especially in the tropicsthat is associated with thermal tides. This is observed in all model runs with the IFS.

\section{b. Diagnostics}

\section{1) RESIDUAL MEAN MERIDIONAL CIRCULATION}

The transformed Eulerian mean framework is used to diagnose the residual mean meridional circulation (Andrews et al. 1987). The residual mean mass streamfunction $\Psi$ is

$$
\Psi \equiv-\frac{\cos \phi}{g} \int_{p}^{0} \bar{v}^{*}\left(\phi, p^{\prime}\right) d p^{\prime}
$$

where the residual meridional velocity $\bar{v}^{*}$ is

$$
\bar{v}^{*}=\bar{v}-\frac{\partial}{\partial p}\left(\frac{\overline{v^{\prime} \theta^{\prime}}}{\partial \bar{\theta} / \partial p}\right)
$$

with $\overline{(.)}$ denoting the zonal mean and ()$^{\prime}$ denoting the deviation of a field from the zonal mean, $v$ is meridional velocity, $\theta$ is potential temperature, $p$ is pressure, $\phi$ is latitude, $g$ is gravitational acceleration, and at $p=0$, $\Psi=0$ is imposed.

\footnotetext{
${ }^{1}$ It should be noted that in the operational IFS cycle 43R1 the launch spectrum is reduced in amplitude by $25 \%$ in the tropics.
} 
To diagnose the contributions of OGWD and NOGWD (recall these refer to the parameterized waves) and the resolved wave drag in driving the residual mean meridional circulation, the downwardcontrol principle of Haynes et al. (1991) is used. It expresses the steady residual mean meridional circulation as a response to drag from breaking/saturating waves aloft.

The downward-control streamfunction $\Psi_{\mathrm{DC}}$ is

$$
\Psi_{\mathrm{DC}} \equiv \frac{\cos \phi}{g} \int_{p}^{0} \frac{\bar{D}\left(\phi, p^{\prime}\right)}{f-(a \cos \phi)^{-1} \partial(\bar{u} \cos \phi) / \partial \phi} d p^{\prime},
$$

where $a$ is Earth's radius, $f$ is the Coriolis parameter, $u$ is zonal wind, and $\bar{D}$ is the zonal-mean wave drag composed of the tendency terms in the zonal momentum equation resulting from the resolved wave drag and NOGWD and OGWD. Resolved wave drag is given by $\nabla \cdot \mathbf{F} / a \cos \phi$, where $\mathbf{F}$ is the Eliassen-Palm (EP) flux:

$$
\mathbf{F}=\left(F_{\phi}, F_{p}\right)=a \cos \phi\left(\frac{\overline{\theta^{\prime} v^{\prime}} \partial \bar{u} / \partial p}{\partial \bar{\theta} / \partial p}-\overline{u^{\prime} v^{\prime}}, \frac{\overline{\theta^{\prime} v^{\prime}}}{\partial \bar{\theta} / \partial p}\left[f-\frac{1}{a \cos \phi} \frac{\partial \bar{u} \cos \phi}{\partial \phi}\right]-\overline{u^{\prime} \omega^{\prime}}\right)
$$

where $\omega$ is the vertical "pressure" velocity. Note that $\nabla \cdot \mathbf{F} / a \cos \phi$ includes orographic and nonorographic gravity wave drag by waves directly resolved by the dynamical core. The effective horizontal resolution, inferred from the kinetic energy spectrum in the lower stratosphere, is up to total wavenumber $\sim 80$.

The residual vertical velocity $\bar{w}^{*}$ is computed following McLandress and Shepherd (2009a):

$$
\bar{w}^{*}=\frac{g H}{p a \cos \phi} \frac{\partial \Psi}{\partial \phi}
$$

where $H$ is the pressure-scale height $H=7 \mathrm{~km}$. Similarly, $\bar{w}_{\mathrm{DC}}^{*}$ can be calculated from $\Psi_{\mathrm{DC}}$.

The vertical mass flux across a pressure surface poleward of latitude $\phi$ in the $\mathrm{NH}$ and $\mathrm{SH}$ is given by Holton (1990) as follows:

$$
F_{\mathrm{NH}}=2 \pi a^{2} \rho \int_{\phi}^{\pi / 2} \bar{w}_{\mathrm{DC}}^{*} \cos \phi d \phi
$$

and

$$
F_{\mathrm{SH}}=2 \pi a^{2} \rho \int_{-\pi / 2}^{\phi} \bar{w}_{\mathrm{DC}}^{*} \cos \phi d \phi
$$

where $\rho$ is density. Instead of evaluating the integral in Eq. (3) on constant angular momentum contours, it is evaluated at a constant latitude. This is a good approximation outside the tropics. Expressed in terms of $\Psi_{\mathrm{DC}}$ and noting that $\Psi_{\mathrm{DC}}$ vanishes at the poles, the downward mass flux poleward of latitude $\phi$ is given by $F_{\mathrm{NH}}=$ $2 \pi a \Psi_{\mathrm{DC}}(\phi)$ and $F_{\mathrm{SH}}=-2 \pi a \Psi_{\mathrm{DC}}(\phi)$. The upward tropical mass flux between two latitudes $\phi$ and $-\phi$ is given by $F_{\mathrm{TR}}=2 \pi a\left[\Psi_{\mathrm{DC}}(\phi)-\Psi_{\mathrm{DC}}(-\phi)\right]$. Here $F_{\mathrm{TR}}$ is calculated between the "turnaround" latitudes as in McLandress and Shepherd (2009a) and Butchart et al. (2011).
The turnaround latitudes are located between the minimum and maximum values of $\Psi_{\mathrm{DC}}$ (i.e., where the tropical upwelling changes to extratropical downwelling).

\section{2) STRATOSPHERIC SUDDEN WARMINGS}

For the free-running setup, composites of SSWs are constructed. Diagnostics similar to those described in McLandress and Shepherd (2009b) are used to identify SSWs. In particular, an SSW is said to occur when the daily mean zonal-mean zonal wind at $10 \mathrm{hPa}$ and $60^{\circ} \mathrm{N}$ becomes easterly between November and March. The date at which this occurs is referred to as the central date. Final vortex breakdowns are excluded by requiring that following the SSW, the zonal wind must become westerly for at least 10 days before the end of April. To avoid counting the same SSW twice, the central dates must be separated by at least 60 days.

\section{3) FinAl WARMing IN THE SH}

The Black and McDaniel (2007) method is used to diagnose the final warming date in the $\mathrm{SH}$. In particular, a final warming occurs when the zonal-mean zonal wind at $60^{\circ} \mathrm{S}$ falls below $10 \mathrm{~m} \mathrm{~s}^{-1}$ and does not return to values above $10 \mathrm{~m} \mathrm{~s}^{-1}$ before the next winter.

\section{c. Evaluation datasets}

To evaluate the nudged runs during the 2006 PJO event, version 3.3 of the temperature product from the Microwave Limb Sounder (MLS) instrument (Livesey et al. 2011) on board the Aura satellite is used between December 2005 and May 2006. MLS has provided continuous observations of the middle atmosphere from September 2004 to the present day. The useful pressure range for the temperature observations is $261-0.001 \mathrm{hPa}$. The vertical resolution of MLS data is $5 \mathrm{~km}$. In addition, 

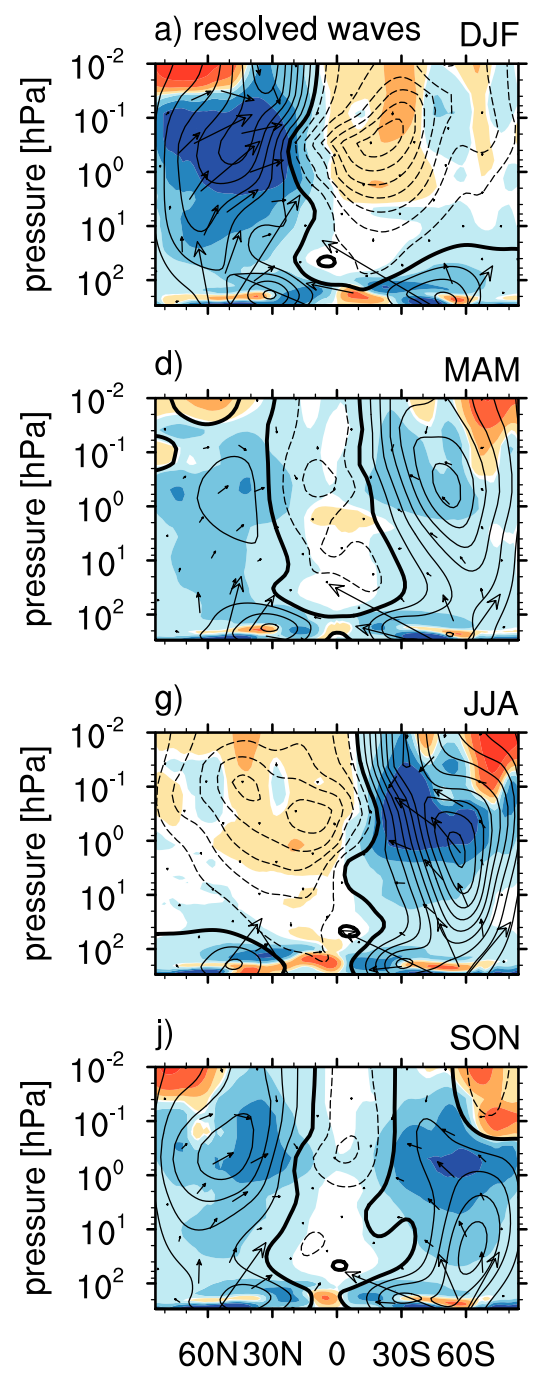

latitude
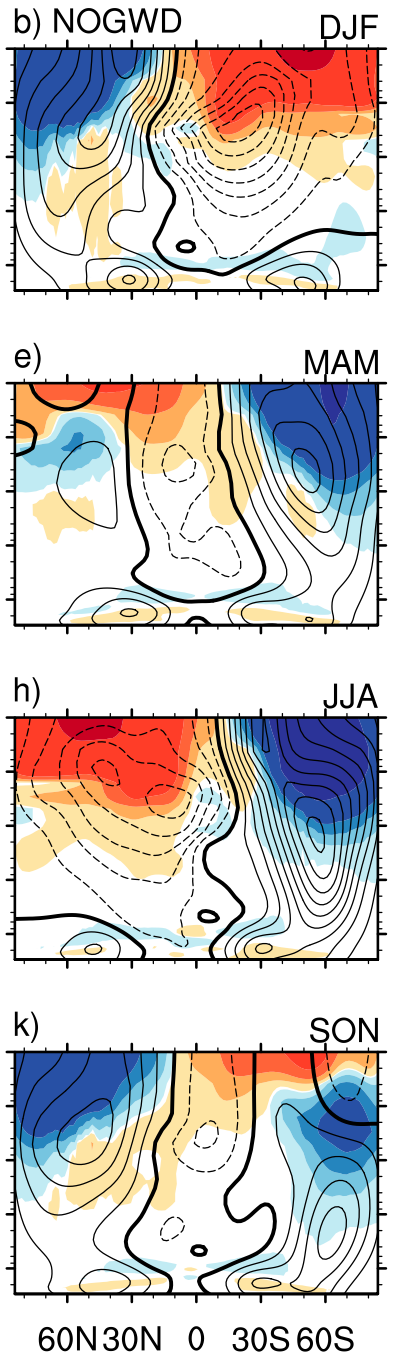

latitude
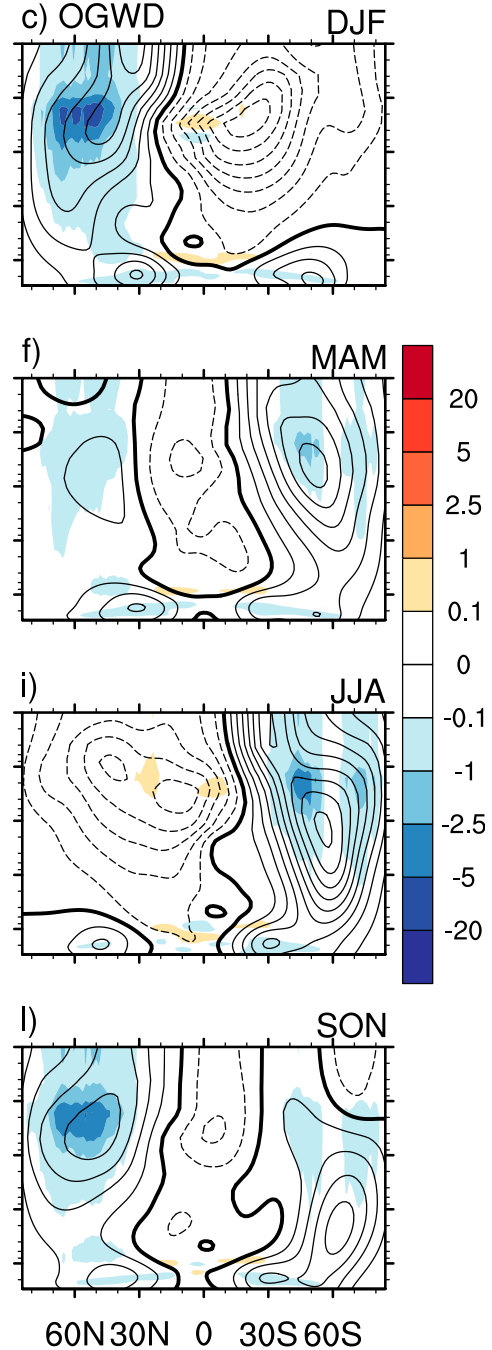

latitude

FIG. 1. Latitude-pressure cross sections of zonal-mean zonal wind tendencies $\left(\mathrm{m} \mathrm{s}^{-1} \mathrm{day}^{-1}\right)$ for the control run in the middle atmosphere: (a)-(c) December-February, (d)-(f) March-May, (g)-(i) June-August, and (j)-(l) September-November. (left) Resolved wave tendency, (center) NOGWD tendency, and (right) OGWD tendency. Negative values are in blue and positive in red. The EP flux vectors are represented by the arrows $\left(\mathrm{m}^{3} \mathrm{~s}^{-2}\right)$. Note the nonlinear contour interval for tendencies. The zonal-mean zonal wind $\left(\mathrm{m} \mathrm{s}^{-1}\right)$ is shown in black contours (contour interval $10 \mathrm{~m} \mathrm{~s}^{-1}$ ), negative contours are dashed and the zero contour is drawn with double thickness. Negative tendencies denote westward momentum deposition and positive tendencies denote eastward momentum deposition.

gradient wind balance zonal winds derived from MLS temperature data are used for evaluation.

The SSW and the SH final warming date statistics in the free-running model are evaluated against the ERAInterim reanalysis statistics.

\section{Zonal momentum budget in the control run}

Before diagnosing the BDC, it is useful to document the distribution of parameterized and resolved wave drag in the middle atmosphere for this version of the IFS. The momentum budget for the IFS at TL159L91 resolution has been diagnosed and discussed for July and December by Orr et al. (2010). The momentum budget at TL255L137 resolution is shown in Fig. 1 for different seasons for the control run. The key features are as follows:

- The zonal wind tendency caused by resolved planetary waves (in shading, first column) and stationary parameterized OGWs (in shading, third column) reflects the fact that these waves can only propagate and break/ saturate in the middle atmosphere when the background zonal winds are westerly. Zonal wavenumber 
decomposition shows that most of the resolved wave drag is coming from wavenumbers 1-3 (not shown). This is true even in the mesosphere as the strong sponge applied above $1 \mathrm{hPa}$ is very effective in damping the higher-frequency smaller-scale resolved waves. The resolved wave drag is stronger in the NH. It is maximal in the $\mathrm{NH}$ in midwinter, but in the $\mathrm{SH}$ in late winter/ spring. This temporal asymmetry is consistent with observations (e.g., Randel 1988; Quintanar and Mechoso 1995) and the theory of Charney and Drazin (1961), which states that planetary waves can propagate into the middle atmosphere when the background westerlies are less than a threshold value. This value is generally below the SH midwinter westerly wind speed.

- In the tropical lower stratosphere, the resolved wave drag consists mostly of synoptic and transient planetary wave breaking on the equatorward flank of the subtropical jet. These waves break throughout the year and are important in driving the tropical upwelling (Randel et al. 2008).

- NOGWs (in shading, second column) are filtered by the background zonal wind: the westward-propagating waves are filtered by the easterlies and the eastwardpropagating waves are filtered by the westerlies (e.g., Shepherd 2000) leading to eastward drag and polar ascent in the summer mesosphere and westward drag and polar descent in the winter mesosphere. In the summer hemisphere, NOGWD dominates the mesospheric drag as the resolved gravity waves are removed by the strong sponge before they reach the mesosphere.

- NOGWD is largest in the SH, where it is the dominant parameterized wave forcing, because of stronger preferential filtering of eastward- versus westwardpropagating waves. In contrast to what is found in lower-resolution models, OGWD is only stronger than NOGWD during the NH winter in the lower mesosphere. The integrand in Eq. (3) is density weighted, so the waves exerting drag at altitudes farther above the stratosphere have less impact on the BDC. Given the above, the effect of the NOGWD flux changes on the BDC, and in particular on the downwelling over the pole, is expected to be smaller in the $\mathrm{NH}$ winter than in the $\mathrm{SH}$ winter.

\section{Results: Residual mean meridional circulation}

\section{a. The control run: Time-mean circulation}

Figure 2 shows the annual-mean tropical upward mass flux (Fig. 2a) and the extended winter mean (OctoberMay for the $\mathrm{NH}$ and March-November for the $\mathrm{SH}$ ) downward mass flux over (Fig. 2b) the NH and (Fig. 2c) the SH polar caps for the control run. The extended winter period comprises all the months for which polar cap downwelling occurs. Both the total downwardcontrol mass flux and the parameterized wave contribution are shown. The downward-control streamfunction and the direct streamfunction [i.e., Eq. (1)] disagree slightly over the extended SH winter pole because of the transience of the vortex breakdown process (not shown).

Table 1 summarizes the resolved and parameterized wave partitioning in driving the tropical upwelling and extended winter polar cap downwelling in both hemispheres. At $70 \mathrm{hPa}$, parameterized waves account for $7 \%$ of the total upwelling (5\% OGWD and $2 \%$ NOGWD) decreasing to $0 \%(2.4 \%$ OGWD and $-2.4 \%$ NOGWD) at $10 \mathrm{hPa}$. These figures should be compared to the multimodel intercomparison of Butchart et al. (2011) where, on average, parameterized waves account for $28 \%$ of the upwelling $(21.1 \%$ OGWD and $7.1 \%$ NOGWD) at $70 \mathrm{hPa}$ and $25.6 \%$ (4.7\% OGWD and $10.9 \%$ NOGWD) at $10 \mathrm{hPa}$. Given the higher horizontal resolution of the IFS compared to the models of Butchart et al. (2011) it is not surprising that the role of parameterized wave drag is smaller in the IFS than in these studies. Note that the relative role of parameterized waves in driving the upwelling increases as one approaches the troposphere in Fig. 2a. This is a result of the NOGWs being launched at $450 \mathrm{hPa}$ and the westward-propagating NOGWs breaking at the critical levels in the subtropics, where the subtropical jets terminate. Hence, the location of the NOGW launch level is likely to impact the parameterized waves that contribute to the tropical upwelling.

There are large differences in the parameterized wave downwelling magnitudes between the hemispheres. At $70 \mathrm{hPa}$, parameterized waves account for only $7 \%$ (all OGWD) of the total extended NH winter pole downwelling, while in the $\mathrm{SH}$ the similar figure is $19 \%$. In the $\mathrm{SH}$ all of the parameterized downwelling is coming from NOGWD. This is expected from Fig. 1, which shows a much larger influence of NOGWD in the SH than in the $\mathrm{NH}$. Generally, the ratio of the parameterized to resolved wave drag in driving the upwelling/downwelling decreases slightly with altitude in the tropics, and increases with altitude over the poles (see Table 1). The parameterized wave downwelling starts to dominate the resolved wave downwelling above $5 \mathrm{hPa}$ in the $\mathrm{SH}$ and above $1 \mathrm{hPa}$ in the $\mathrm{NH}$.

\section{b. Sensitivity to nonorographic gravity wave drag: Time-mean circulation}

Given the importance of NOGWD at higher resolution, the sensitivity of tropical upwelling and polar cap 
a) control

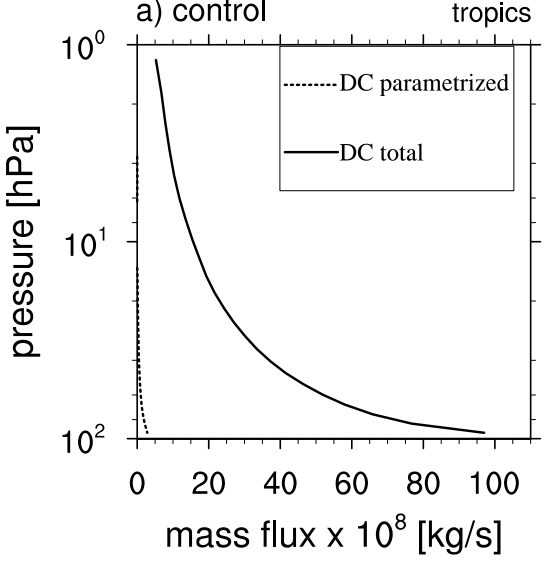

d)

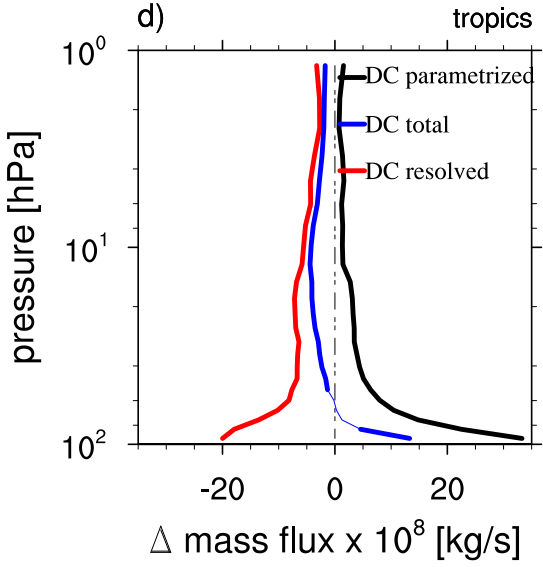

b) control

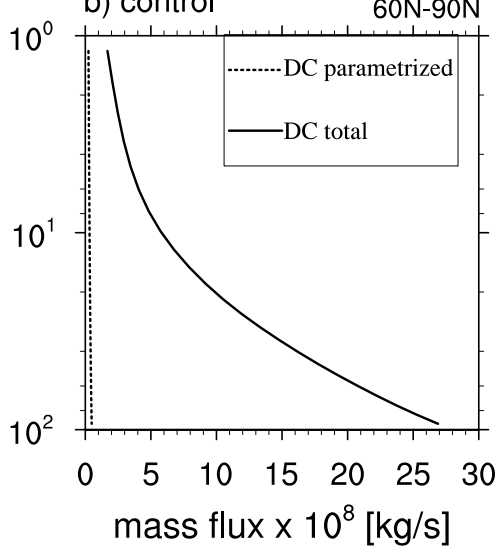

e)

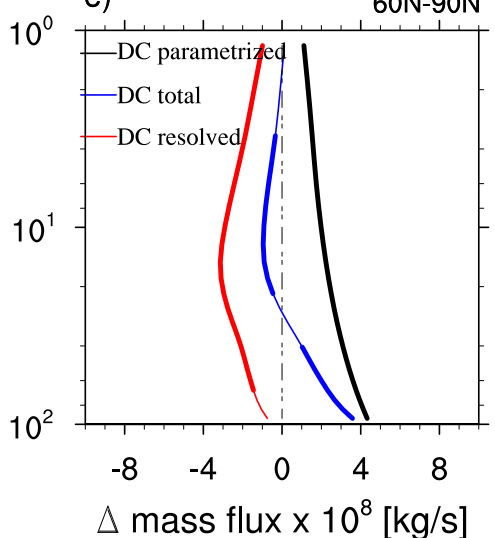

c) control

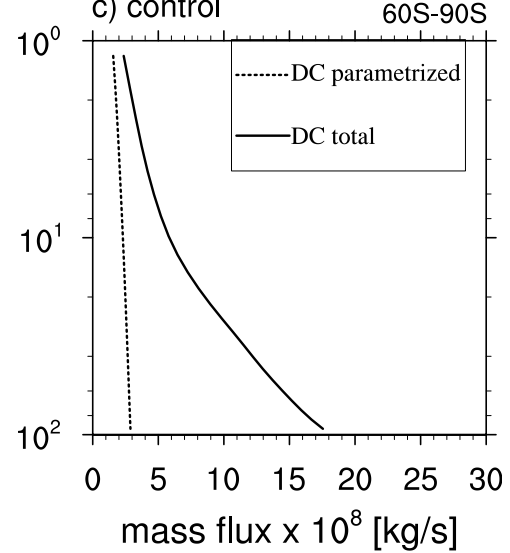

f)

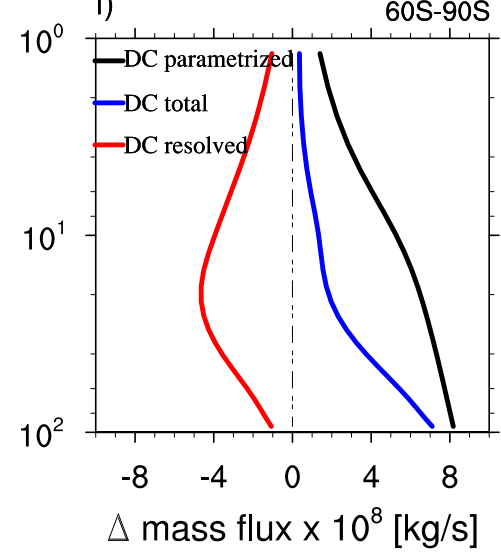

FIG. 2. (a) Annual-mean upward mass flux over the tropics and extended winter downward mass flux over (b) the NH (October-May) and (c) the SH (March-November) polar cap (poleward of $60^{\circ} \mathrm{N} / \mathrm{S}$ ) for the control run. The solid lines show the total downward-control mass flux and the dashed lines show the parameterized wave contribution. (d)-(f) Difference in the mass fluxes between increased and reduced NOGWD runs. The thickened lines in (d)-(f) show regions where the response is significant at the $95 \%$ level by the Student's $t$ test on the means. Mass flux calculated from the DC streamfunction (blue), from the parameterized wave contribution to the DC streamfunction (black), and from the resolved wave contribution to the DC streamfunction (red).

downwelling to changes in NOGWD flux is now examined. Table 1 summarizes the changes to resolved and parameterized wave partitioning brought about by a decrease in NOGWD flux by 3.75 times and an increase in NOGWD flux by 3.75 times. As expected, the parameterized wave driving decreases (increases) with a decrease (increase) in NOGWD flux. For example, at $70 \mathrm{hPa}$, the parameterized wave contribution to the tropical upwelling and $\mathrm{NH}$ polar cap downwelling reduces to $2 \%$ with a reduction in NOGWD flux. Similarly, the parameterized wave contribution to the 70-hPa tropical upwelling and $\mathrm{NH}$ polar cap downwelling increases to nearly $20 \%$ with an increase in NOGWD flux. For the SH polar cap downwelling, the corresponding figure is $6 \%$ for a decrease in NOGWD flux and $45 \%$ for an increase in NOGWD flux.
Figure 2 shows the difference in (Fig. 2d) the annualmean tropical upward mass flux and (Fig. 2e) the extended NH and (Fig. 2f) SH winter downward mass flux between the increased and reduced NOGWD runs. As expected from the dominance of NOGWD in the $\mathrm{SH}$, varying NOGWD flux has the most impact there. In particular, the total downwelling (blue for the downward-control streamfunction) increases in response to increase in NOGWD (see Table 1). For example, increasing NOGWD flux from the control value by 3.75 times leads to a $\sim 30 \%$ increase in the $\mathrm{SH}$ polar cap downwelling at $70 \mathrm{hPa}$. The net effect of the increased downwelling is to warm the SH stratospheric winter pole by $\sim 15 \mathrm{~K}$ (not shown). However, the response in the total downwelling is not directly proportional to the change in NOGWD-induced downwelling (black lines) as the resolved wave downwelling (red lines) opposes the 
TABLE 1. Resolved and parameterized (OGWD and NOGWD) wave contribution (\% of the total) to the annual-mean tropical mass flux and extended winter (March-November for the SH, and October-May for the NH) polar cap downward mass flux for the control, reduced NOGWD, and increased NOGWD runs at $10 \mathrm{hPa}$ and at $70 \mathrm{hPa}$. Positive percentage denotes tropical upwelling and polar cap downwelling and negative percentage denotes tropical downwelling and polar cap upwelling.

\begin{tabular}{|c|c|c|c|c|c|c|c|}
\hline \multirow[b]{2}{*}{ Expt } & \multirow[b]{2}{*}{ Region } & \multirow[b]{2}{*}{ Pressure $(\mathrm{hPa})$} & \multicolumn{3}{|c|}{$\begin{array}{l}\text { Parameterized wave drag } \\
(\%)\end{array}$} & \multirow{2}{*}{$\begin{array}{c}\text { Resolved } \\
\text { wave drag (\%) }\end{array}$} & \multirow{2}{*}{$\begin{array}{c}\text { Mass flux } \\
\left(\times 10^{8} \mathrm{~kg} \mathrm{~s}^{-1}\right)\end{array}$} \\
\hline & & & OGWD & NOGWD & All & & \\
\hline \multirow{6}{*}{ Control } & Annual-mean upwelling & 10 & 2.4 & -2.4 & 0 & 100 & 15.5 \\
\hline & & 70 & 5 & 2 & 7 & 93 & 58.1 \\
\hline & NH polar cap downwelling & 10 & 14 & 5 & 19 & 81 & 5.7 \\
\hline & & 70 & 7 & 0 & 7 & 93 & 22.2 \\
\hline & SH polar cap downwelling & 10 & 0 & 40.6 & 40.6 & 59 & 5.8 \\
\hline & & 70 & 0 & 19 & 19 & 81 & 15 \\
\hline \multirow[t]{6}{*}{ Reduced NOGWD } & Annual-mean upwelling & 10 & 2.5 & -3 & -0.5 & 100.5 & 16.1 \\
\hline & & 70 & 5 & -3 & 2 & 98 & 57.1 \\
\hline & $\mathrm{NH}$ polar cap downwelling & 10 & 14 & -9 & 5 & 95 & 6.2 \\
\hline & & 70 & 8 & -6 & 2 & 98 & 20.7 \\
\hline & SH polar cap downwelling & 10 & 0 & 12 & 12 & 88 & 5.4 \\
\hline & & 70 & 0 & 6 & 6 & 94 & 13.5 \\
\hline \multirow[t]{6}{*}{ Increased NOGWD } & Annual-mean upwelling & 10 & 4 & 6 & 10 & 90 & 11.8 \\
\hline & & 70 & 4 & 16 & 20 & 80 & 57.4 \\
\hline & $\mathrm{NH}$ polar cap downwelling & 10 & 7.5 & 38.5 & 46 & 54 & 5.2 \\
\hline & & 70 & 5 & 14 & 19 & 81 & 23.2 \\
\hline & SH polar cap downwelling & 10 & 0 & 88 & 88 & 12 & 6.7 \\
\hline & & 70 & 0 & 45 & 45 & 55 & 19.3 \\
\hline
\end{tabular}

NOGWD changes in the time mean. Interestingly, in the $\mathrm{NH}$ polar mid- and upper stratosphere and in the tropics, the decrease in the resolved wave driving in response to the increase in NOGWD leads to a decrease in total downwelling (see Table 1).

To understand the changes in the resolved wave forcing, Figs. 3a,b show the difference in the extended $\mathrm{NH}$ and SH winter stratospheric EP flux and its divergence between the increased and reduced NOGWD runs. The resolved wave drag corresponds to EP flux convergence; hence, the red regions indicate less resolved wave drag when NOGWD is increased. Over the polar vortex, the resolved wave response falls into two distinct regions: an increase in the resolved wave drag in the lower stratosphere and a decrease in the resolved wave drag in the mid- to upper stratosphere. This is reflected in the vertical profile of the response of the downwelling driven by the resolved waves in Figs. 2e and $2 \mathrm{f}$ (red lines).

To quantify the response in the resolved waves in the lower and upper stratosphere, an EP-flux budget (following Kushner and Polvani 2004) is constructed for two boxes in the vicinity of the polar vortex between $35^{\circ} \mathrm{N} / \mathrm{S}$ and $90^{\circ} \mathrm{N} / \mathrm{S}: 1$ ) a lower-stratospheric box from 70 to $10 \mathrm{hPa}$ and 2) an upper-stratospheric/lower-mesospheric box from 10 to $0.1 \mathrm{hPa}$. The budget is shown for the increased (red) and reduced (green) NOGWD runs in Figs. $3 \mathrm{c}$ and $3 \mathrm{~d}$. In the winter lower stratosphere there is
$5 \%$ more wave drag in the $\mathrm{NH}$ and $25 \%$ more wave drag in the SH in response to increased NOGWD. This likely occurs as a result of a weakened vortex-brought about by the increase in NOGWD-that is more amenable to wave breaking lower down. There is a marked reduction in the resolved waves entering $(20 \%$ less in the $\mathrm{NH}$ and $25 \%$ less in the $\mathrm{SH}$ ) and breaking (63\% less wave breaking in the $\mathrm{NH}$ and $90 \%$ less wave breaking in the $\mathrm{SH})$ in the mid- to upper stratosphere.

In summary, increasing NOGWD weakens the polar night jet and thereby decreases resolved wave propagation into the polar mid- to upper stratosphere during the extended winter season, leading to less resolved wave breaking there. This counteracts the polar cap downwelling increase by the NOGWD such that the total mid- to upper-stratospheric downwelling decreases in the $\mathrm{NH}$ and increases in the $\mathrm{SH}$ in response to increase in NOGWD. In the lower stratosphere the polar cap downwelling increases in both hemispheres as the resolved waves reinforce the NOGWD perturbation.

\section{c. The control run: The seasonal cycle}

To understand how the partitioning of parameterized and resolved waves in driving the polar cap downwelling differs between seasons and between the hemispheres, it is useful to examine the seasonal cycle of the polar cap average $\bar{w}_{\mathrm{DC}}^{*}$ Figure 4 shows the seasonal cycle of polar cap average $\bar{w}_{\mathrm{DC}}^{*}$ (thick solid lines, top panels) and its 

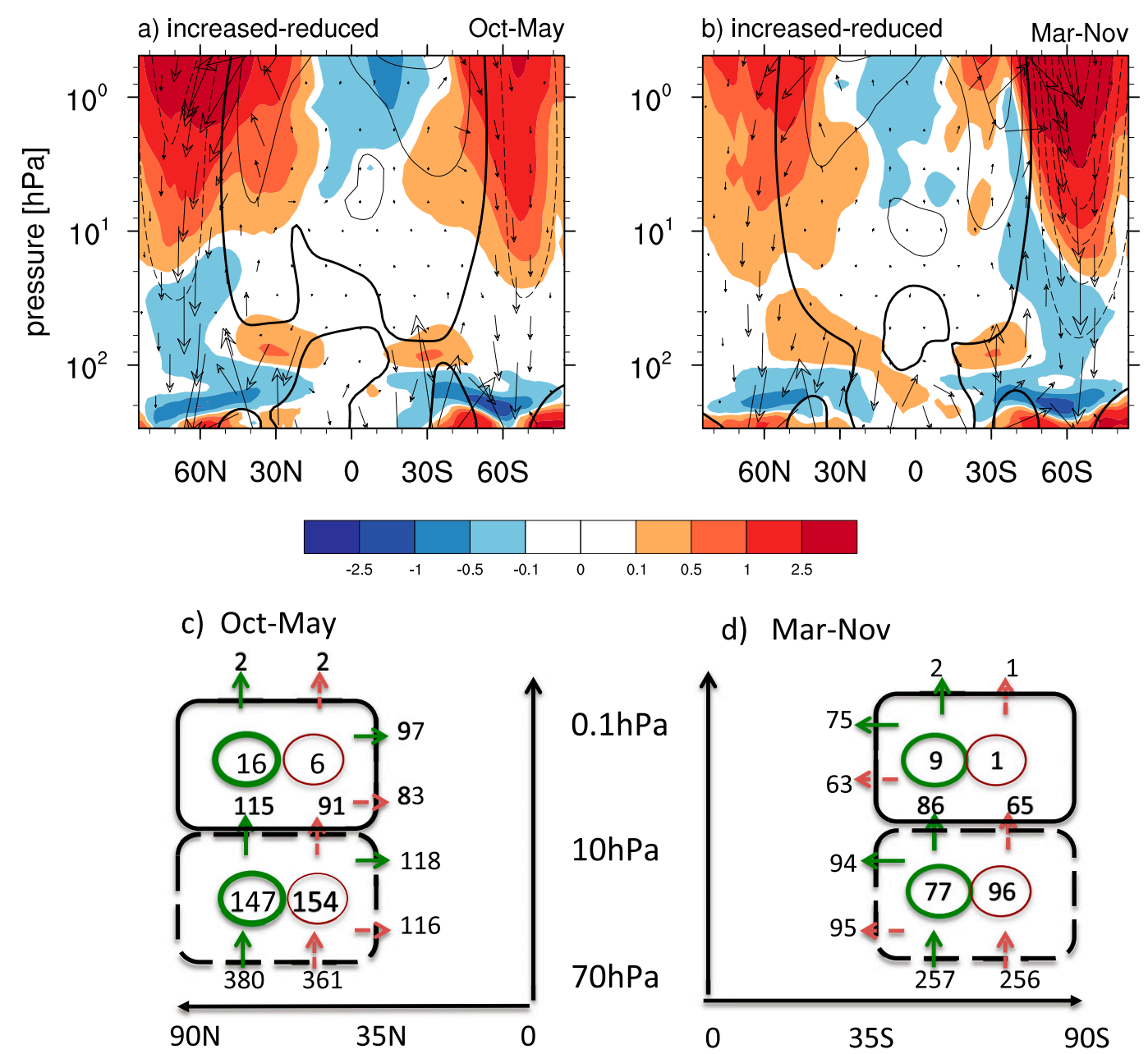

FIG. 3. (a),(b) Latitude-pressure cross sections of the resolved wave drag difference (shading; $\mathrm{m} \mathrm{s}^{-1} \mathrm{day}^{-1}$ ) between the increased and reduced NOGWD runs for the extended (a) NH (October-May) and (b) SH (MarchNovember) winters. The EP flux vectors are shown by the arrows $\left(\mathrm{m}^{3} \mathrm{~s}^{-2}\right)$. The zonal-mean zonal wind difference $\left(\mathrm{m} \mathrm{s}^{-1}\right.$ ) is shown in black contours (contour interval $10 \mathrm{~m} \mathrm{~s}^{-1}$ ), negative contours are dashed, and the zero contour is drawn with double thickness. (c),(d) The EP flux budget $\left(10^{16} \mathrm{~N} \mathrm{~m}\right.$ ) for the extended (c) NH and (d) SH winters for the reduced NOGWD (green) and increased NOGWD (red) run for the two boxes (see text). The positive numbers inside the boxes show the net resolved wave convergence (i.e., the wave breaking). The vertical arrows represent vertical EP fluxes and the horizontal arrows represent horizontal EP fluxes.

parameterized wave (dashed lines, bottom panels) and resolved wave (thin solid lines, bottom panels) contribution for the control simulation (black lines).

The upwelling in the summer mesosphere is mostly driven by NOGWD over both poles with little contribution from the resolved waves (not shown explicitly, but compare first and second columns of Fig. 1). In the $\mathrm{NH}$, the downwelling is maximum in midwinter in January and is predominantly driven by resolved waves in the stratosphere (apart from the upper stratosphere where the parameterized waves dominate the downwelling in autumn). In the NH the parameterized wave downwelling is maximum during the stratospheric zonal wind maximum in the late autumn/early winter, whereas the maximum in the resolved wave downwelling is offset slightly in time. In contrast, in the SH the downwelling is maximal in the spring season and the time of maximum downwelling occurs later as one descends through the stratosphere. The resolved waves dominate the downwelling in the spring season, whereas the parameterized waves dominate the downwelling in midwinter in the mid to upper stratosphere (see also Fig. 1), at the time of maximum westerlies. This seasonal behavior of the resolved and parameterized waves is consistent with observations (e.g., Randel 1988; Quintanar and Mechoso 1995; Pulido and Thuburn 2008) and also observed in CMAM (Shaw et al. 2009). The different timing in the resolved and parameterized wave downwelling will be 

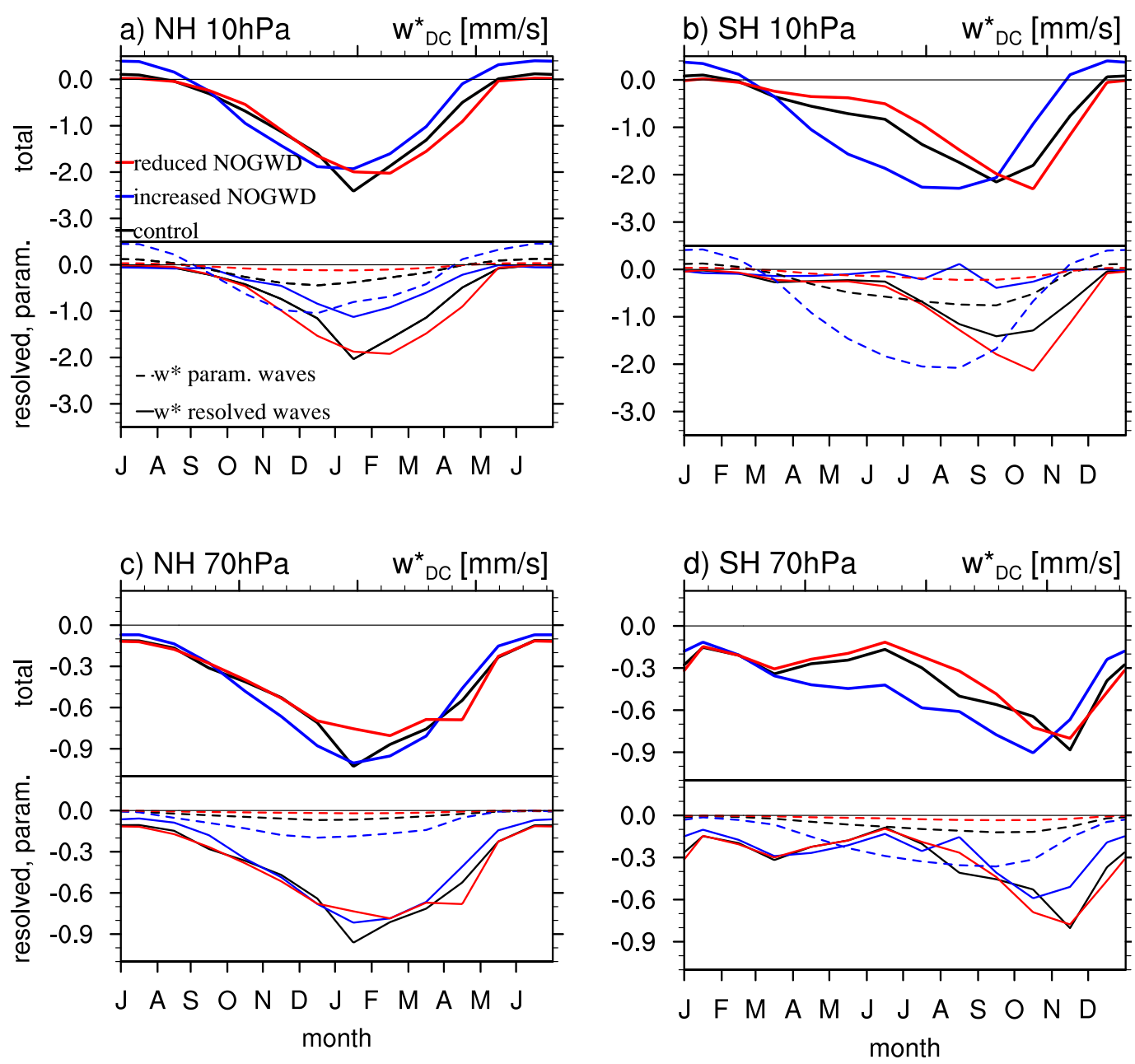

FIG. 4. Seasonal cycle of the downward-control residual vertical velocity $\bar{w}_{\mathrm{DC}}^{*}$ (thick lines, top panels in each figure), split into its parameterized wave (dashed lines, bottom panels in each figure) and resolved wave (thin solid lines, bottom panels in each figure) contributions averaged over the (a),(c) NH and (b),(d) SH polar cap (between $60^{\circ}$ and $85^{\circ} \mathrm{N} / \mathrm{S}$ ) at (a),(b) $10 \mathrm{hPa}$ and (c),(d) $70 \mathrm{hPa}$, respectively. Black lines denote the control run, red lines denote the reduced NOGWD run, and blue lines denote the increased NOGWD run. Note that the time axis has been shifted by six months in (a),(c) for clarity.

important for the response in the seasonality of $\bar{w}_{\mathrm{DC}}^{*}$ to changes in NOGWD. Note that unlike in the lowerresolution models, OGWD does not contribute to the polar cap averaged $\bar{w}_{\mathrm{DC}}^{*}$ in the $\mathrm{SH}$ (not shown).

\section{d. Sensitivity to nonorographic gravity wave drag: \\ The seasonal cycle}

The time-mean response might paint a misleading picture of the interaction between the resolved and the parameterized waves as there is a strong seasonality in the BDC forcing. The seasonal cycle of the polar cap average $\bar{w}_{\mathrm{DC}}^{*}$, together with its resolved and parameterized wave driving contributions, is also shown in Fig. 4 for the reduced NOGWD run (in red) and increased NOGWD (in blue). Figure 5 shows the seasonal cycle of the difference in the polar cap average $\bar{w}_{\mathrm{DC}}^{*}$ between the increased NOGWD and reduced NOGWD runs.

In the summer, the total $\bar{w}_{\mathrm{DC}}^{*}$ response in the upper stratosphere is proportional to changes in NOGWD as the easterlies filter out stationary planetary waves and smaller-scale orographic gravity waves, leaving no resolved waves with which to interact (see Fig. 1). Note that the seasonal transition from downwelling to upwelling occurs earlier in the increased NOGWD run, especially in the $\mathrm{SH}$. This appears to be tied in with the onset of the final warming, which occurs earlier in the increased NOGWD run; because the westerlies weaken earlier in the increased NOGWD run, the eastward-propagating NOGWs can propagate into the upper stratosphere and mesosphere 
a) $60 \mathrm{~N}-90 \mathrm{~N} 10 \mathrm{hPa}$

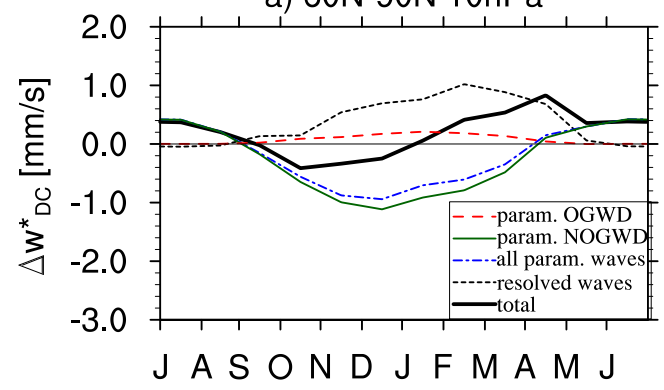

c) $60 \mathrm{~N}-90 \mathrm{~N} 70 \mathrm{hPa}$

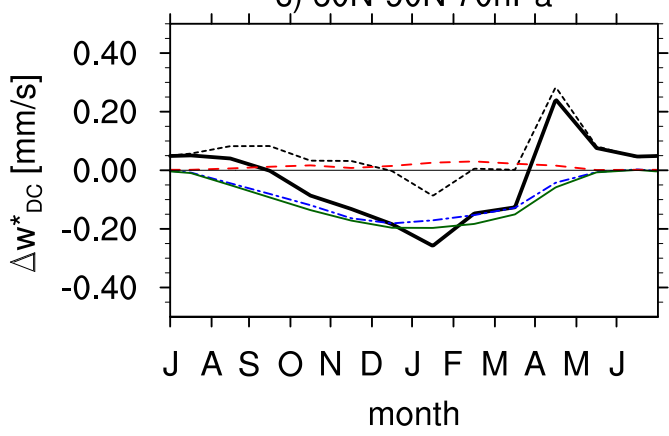

b) $60 \mathrm{~S}-90 \mathrm{~S} 10 \mathrm{hPa}$

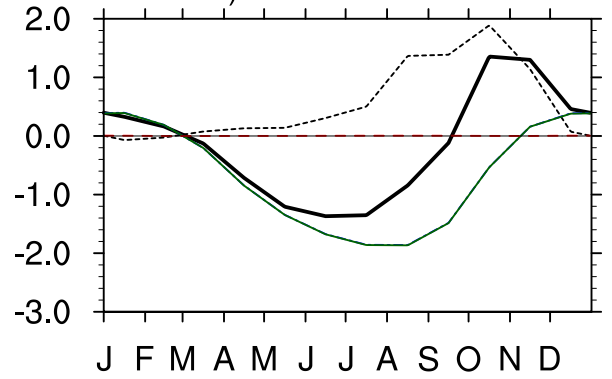

d) $60 \mathrm{~S}-90 \mathrm{~S} 70 \mathrm{hPa}$

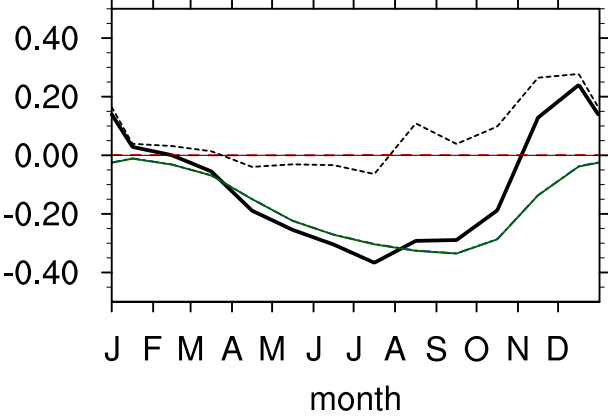

FIG. 5. Seasonal cycle of the difference in the downward-control residual vertical velocity $\bar{w}_{\text {DC }}^{*}$ (thick black lines) between the increased and reduced NOGWD runs, split into its parameterized wave (dashed-dotted blue lines) and resolved wave (dotted black lines) contributions. The NOGWD change is shown in solid green and the OGWD change is shown in dashed red: $\bar{w}_{\mathrm{DC}}^{*}$ response averaged over the (a),(c) $\mathrm{NH}$ and (b),(d) SH polar cap at (a),(b) $10 \mathrm{hPa}$ and at (c),(d) $70 \mathrm{hPa}$. Note that the time axis has been shifted by six months in (a),(c) for clarity.

earlier. When the eastward-propagating waves saturate they induce upwelling (see Fig. 4b).

To examine the effect of NOGWD on the final warming date, Fig. 6 shows the average of the final warming dates in the $\mathrm{SH}$ as a function of pressure together with the ERA-Interim climatology from 2004 to 2015 for reference (thick black dashed-dotted line). As the NOGWD is increased, the climatological final warming date occurs earlier in the stratosphere as the vortex is weakened and is thus more amenable to wave breaking. This is consistent with more resolved wave drag in the lower stratosphere (see Fig. 3b). In the mesosphere, however, the vortex breakdown is delayed when the NOGWD is substantially increased. This is, as discussed above, due to the reduced resolved wave drag entering the upper stratosphere and mesosphere. Resolved wave drag accelerates the seasonal evolution toward easterlies in the spring, so when it is reduced, the seasonal cycle is delayed. Note that the NOGWD tends to drag the zonal winds to zero at mid- to high latitudes near the model top as the waves, originating at $450 \mathrm{hPa}$, are filtered such that only those with phase speeds of opposite sign to the zonal wind are left. Therefore NOGWD does not contribute to the vortex breakdown in the same way as the resolved waves. It should be emphasized that here the NOGWD is reduced via the sources, but the total resolved wave drag is largely unchanged, only its location is altered.

In the NH, OGWD partly compensates for the increase in NOGWD-induced downwelling in winter (cf. dashed red, solid green, and dashed-dotted blue curves in Figs. 5a and 5c). The resolved wave drag shifts vertically in response to increase in the NOGWD-induced downwelling in the mid- to upper stratosphere (cf. dotted lines in Figs. 5a and 5c), but there is a seasonal offset in the resolved wave response. As a result, the increase in net downwelling expected from increased NOGWD transitions to a decrease in downwelling toward the end of the extended winter season, in both the lower and middle stratosphere, and in both hemispheres.

The seasonal offset in the resolved wave response is larger in the $\mathrm{SH}$, where the changes to NOGWD flux significantly modify the seasonal evolution of polar capaveraged $\bar{w}_{\mathrm{DC}}^{*}$. When NOGWD is increased, it has the most impact in midwinter in the $\mathrm{SH}$ when the resolved wave driving is weak in the stratosphere. Hence, the change in the SH total polar cap-averaged $\bar{w}_{\mathrm{DC}}^{*}$ is almost proportional to NOGWD flux changes in midwinter. Increasing NOGWD weakens and shifts the polar night jet equatorward. This leads to less resolved waves 


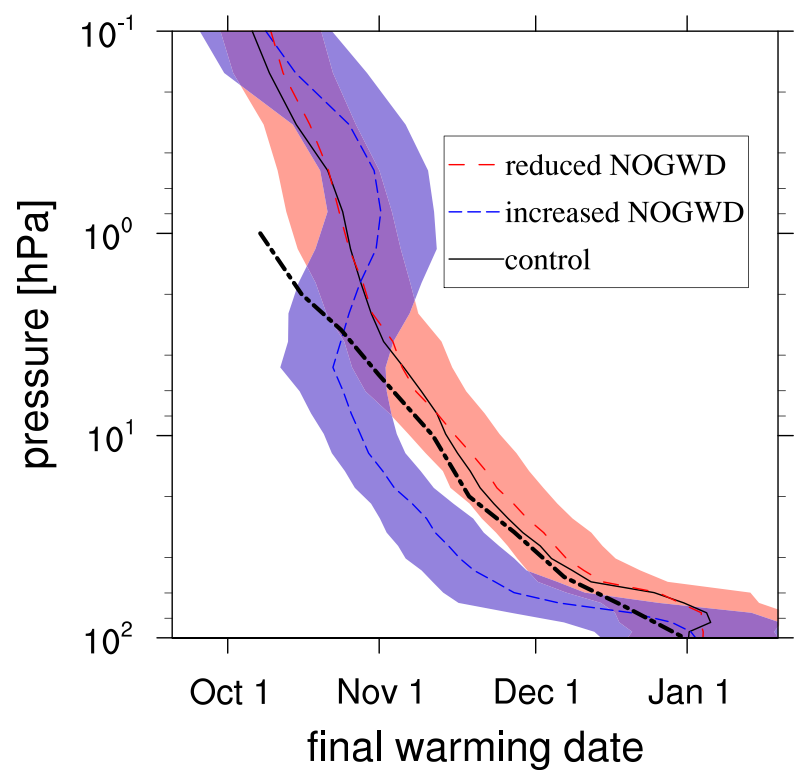

FIG. 6. Average of the final warming dates in the $\mathrm{SH}$ for the control run (solid black), the reduced NOGWD run (long-dashed red), and the increased NOGWD run (short-dashed blue). The average of the ERA-Interim final warming dates between 2004 and 2015 is shown in thick dashed-dotted black contour. The shading shows the $2 \sigma$ interval for the increased and reduced NOGWD runs only.

entering the mid- to upper stratosphere-especially in the SH spring-resulting in less resolved wave downwelling (see Fig. 5). The resolved waves appear to be unable to propagate as high into the stratosphere in the increased NOGWD run. As the parameterized wave downwelling is weak in the spring, the decrease in the resolved wave downwelling dominates and results in a decrease in downwelling with an increase in NOGWD. In the lower stratosphere (Figs. 5c,d), the resolved waves tend to amplify the NOGWD changes in midwinter in both hemispheres, consistent with the increased wave drag in the midlatitude lower stratosphere shown in Figs. 3 and 4.

\section{Results: SSWs}

Having examined the sensitivity of the BDC climatology to the NOGWD flux perturbations in the previous section, the next step is to assess the impact of these perturbations on SSWs, which are important for tropospheric predictability. In what follows the impact of NOGWD changes on the 2006 PJO life cycle is first examined in the nudged setup before discussing the impact of NOGWD on SSWs in general.

\section{a. 2006 PJO event in the nudged model}

Figures $7 \mathrm{a}$ and $7 \mathrm{~b}$ show the evolution of the gradient zonal-mean zonal wind averaged from $50^{\circ}$ to $70^{\circ} \mathrm{N}$ and the polar cap-averaged zonal-mean temperature from MLS. In Figs. $7 \mathrm{c}$ and $7 \mathrm{~d}$ the evolution of $50^{\circ}-70^{\circ} \mathrm{N}$ ensemble-mean zonal-mean zonal wind and the polar cap-averaged zonal-mean temperature is shown for the nudged control run. The nudged control run captures the 2006 PJO life cycle reasonably accurately, albeit the SSW occurs in the model two weeks earlier than in the observations (recall that the nudging is applied only below $500 \mathrm{hPa}$ and the stratosphere evolves freely). That this is not an artifact of the ensemble averaging is shown in Fig. 7i, where the time series of the zonal-mean zonal wind at $60^{\circ} \mathrm{N}$ and $10 \mathrm{hPa}$ is shown for all ensemble members of the control run. It is clear that all the ensemble members predict an earlier onset of the PJO event than what is observed (MLS observations are shown in thick red line). The onset of the SSW is improved if the nudging is carried out below $100 \mathrm{hPa}$. The persistence length (quantified here by the number of days the zonal-mean zonal wind is easterly at $60^{\circ} \mathrm{N}$ and $10 \mathrm{hPa}$ following the central date) of 24 days, however, is the same in the control run and in MLS.

The evolution of a typical long-lived SSW life cycle has been described in detail (e.g., Siskind et al. 2010; Limpasuvan et al. 2012; Tomikawa et al. 2012; Hitchcock and Shepherd 2013; McLandress et al. 2013) and is summarized here for completeness. To aid the description of the life cycle, the ensemble-mean zonal wind tendencies and the residual vertical velocity are shown in Fig. 8 for the nudged control run (in shading, left column). The initial stratospheric warming is a result of enhanced resolved planetary wave drag (see Fig. 8e). As the zonal wind in the stratosphere becomes easterly during the PJO event, the westward-propagating NOGWs, the resolved planetary waves, and the OGWs are no longer able to enter the middle atmosphere. This, together with the transient response that generates an upward closing cell near the upper boundary, results in a weaker residual circulation and the concomitant cooling in the mesosphere [see also Fig. 4 in Ren et al. (2008)]. The middle atmosphere easterlies permit eastward phase speed NOGWs to propagate upward resulting in the net eastward NOGWD. This contributes to the reformation of the polar night jet as the net eastward NOGWD induces upwelling and cooling of the polar regions. Following a PJO event, initially temperature evolves almost entirely diabatically as the resolved and the parameterized stationary orographic gravity wave forcing is suppressed. The descent of mesospheric cooling follows the vertical gradient in the climatological cooling profile and the radiative damping time, which decreases with decreasing pressure [see Figs. 2a and 10 of Hitchcock and Shepherd (2013)]. As the westerlies in the mesosphere recover, 

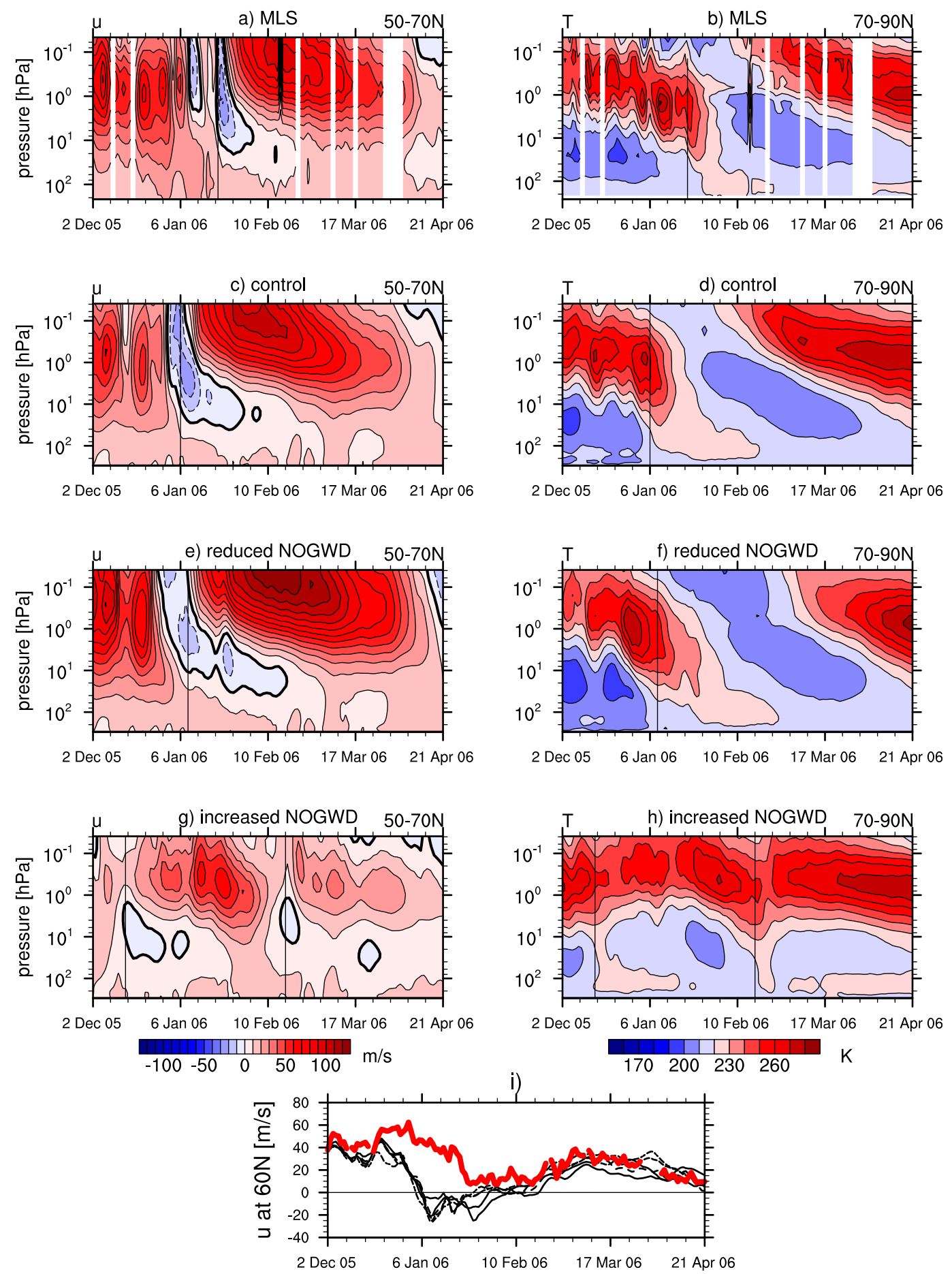

FIG. 7. Pressure-time cross sections of (left) the zonal-mean zonal wind averaged from $50^{\circ}$ to $70^{\circ} \mathrm{N}$ and (right) the polar cap-averaged (from $70^{\circ}$ to $90^{\circ} \mathrm{N}$ ) zonal-mean temperature for the 2006 PJO event. (a),(b) MLS observations (zonal wind computed using gradient wind balance); (c),(d) control nudged run; (e),(f) reduced NOGWD nudged run; and $(\mathrm{g}),(\mathrm{h})$ increased NOGWD nudged run. For the simulations, the ensemble mean is shown. The vertical lines mark the central date of SSWs. (i) Zonal-mean zonal wind at $60^{\circ} \mathrm{N}$ and at $10 \mathrm{hPa}$ for all ensemble members in the control run (black lines) and the MLS observations (thick red line). 
a) NOGWD

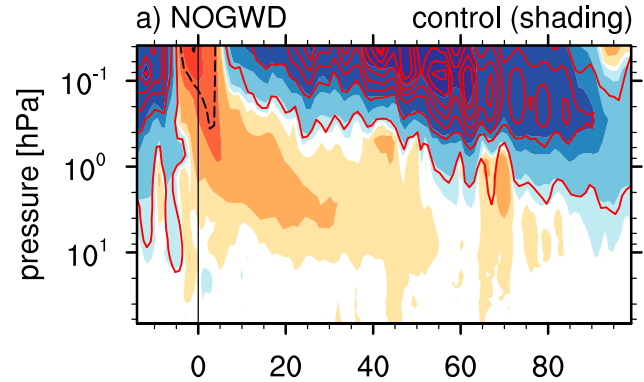

c) OGWD

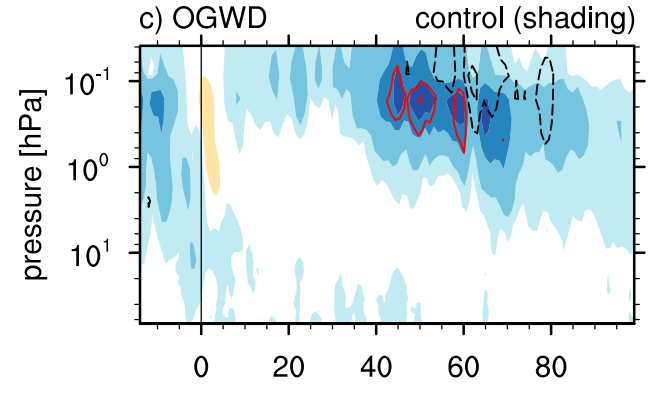

e) resolved

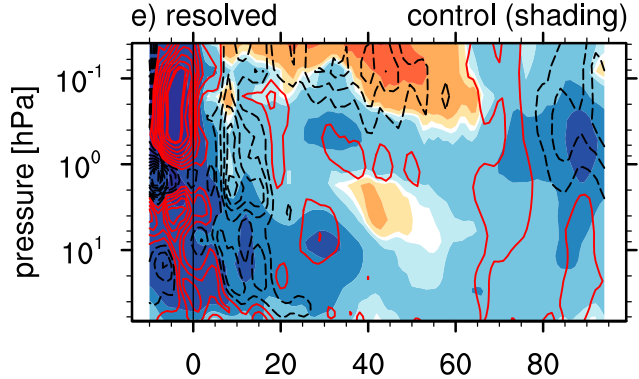

g) $w^{*}$

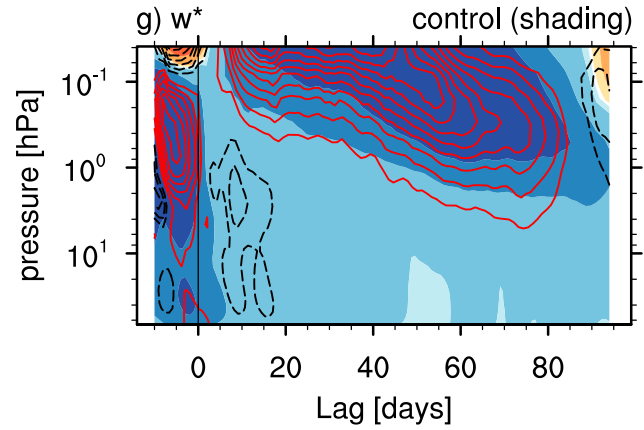

b) NOGWD increased (shading)

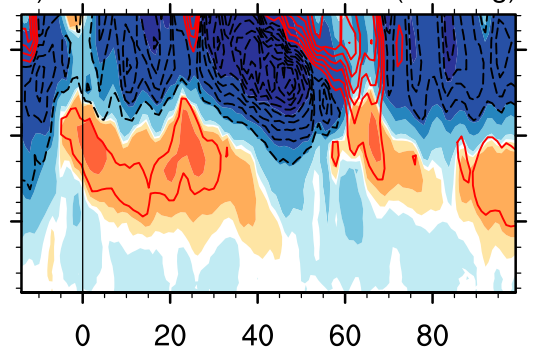

d) OGWD

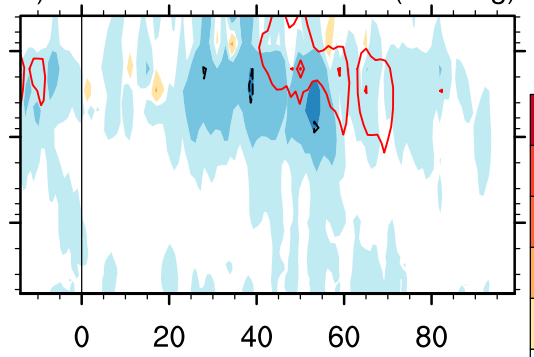

20

5

2.5

0.5

0.1

f) resolved

increased (shading)

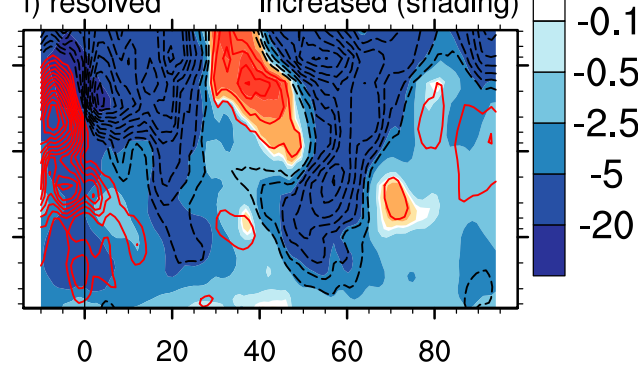

h) $w^{*}$ increased (shading)

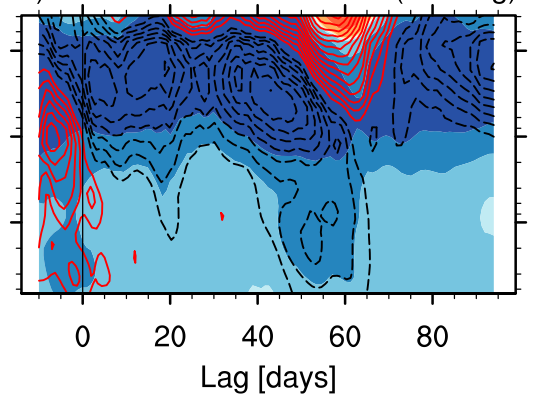

FIG. 8. (shading) Pressure-time cross sections of the ensemble-mean polar cap-averaged (a),(b) NOGWD tendency; (c),(d) OGWD tendency; (e),(f) resolved wave tendency; and (g),(h) residual vertical velocity $\bar{w}^{*}$ for (left) the control run and (right) the increased NOGWD run, during the life cycle of the 2006 PJO event. Dashed black (negative) and solid red (positive) contours: response in tendencies and $\bar{w}^{*}$ to (left) reducing NOGWD and (right) increasing NOGWD [contour interval is (a)-(d) $4 \mathrm{~m} \mathrm{~s}^{-1} \mathrm{day}^{-1}$; (e),(f) $2 \mathrm{~m} \mathrm{~s}^{-1}$ day ${ }^{-1}$; and (g),(h) $1 \mathrm{~mm} \mathrm{~s}^{-1}$ ]. Time zero represents the central dates of SSWs: 6 Jan 2006 for the control run, 9 Jan 2006 for the reduced NOGWD run, and 15 Dec 2005 for the increased NOGWD run. The resolved wave tendency and $\bar{w}^{*}$ are smoothed by taking a 10-day running mean. Note that the pressure range is from 70 to $0.01 \mathrm{hPa}$. 
a) $U$ reduced NOGWD-control

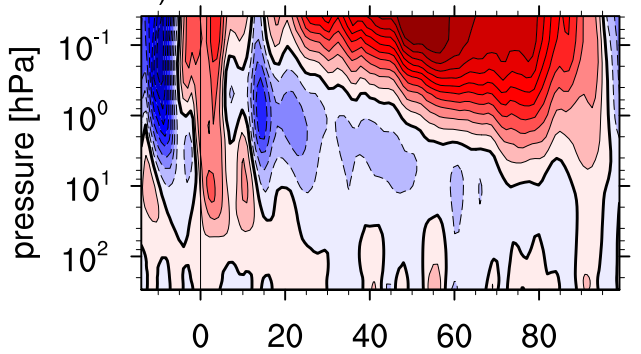

c) $U$ increased NOGWD-control

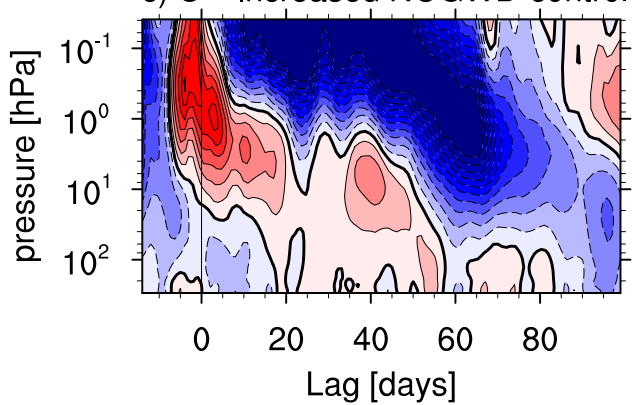

b) $T$ reduced NOGWD-control

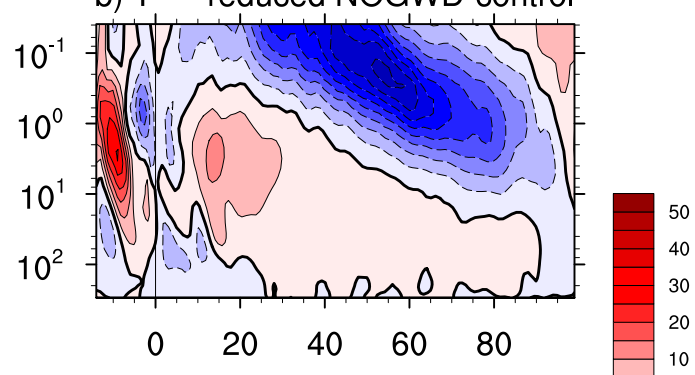

d) $T$ increased NOGWD-control

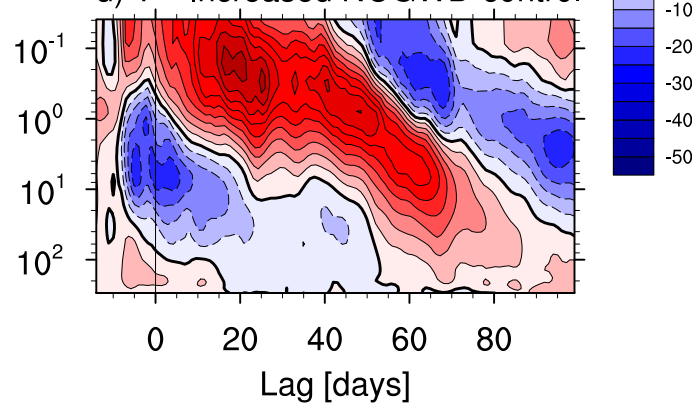

FIG. 9. Difference in (a),(c) the $50^{\circ}-70^{\circ} \mathrm{N}$ zonal-mean zonal wind $\left(\mathrm{m} \mathrm{s}^{-1}\right)$ and (b),(d) the polar cap-averaged zonal-mean temperature $(\mathrm{K})$ between the control run and (a),(b) the reduced NOGWD run and (c),(d) the increased NOGWD run.

the westward-propagating NOGWs are no longer filtered out. On reaching the mesosphere, westward NOGWs induce downwelling and are hence responsible for the reformation of the stratopause, which descends downward with time. The short radiative damping time scales in the mesosphere imply that any temperature anomaly has to be maintained by dynamical heating. Once the stratospheric winds have become westerly throughout the stratosphere, OGWs can propagate into the middle atmosphere and contribute to the reformation of the stratopause by inducing dynamical heating. The persistence of the lower-stratospheric warm anomaly following the PJO event is a result of strongly suppressed wave driving and weak climatological radiative cooling [see Figs. 12 and 10 of Hitchcock and Shepherd (2013)]. This also removes any mechanism for chaotic error growth between the troposphere and stratosphere.

Now the effect of changing NOGWD on the PJO life cycle is examined. Figures $7 \mathrm{e}-\mathrm{h}$ show the evolution of the $50^{\circ}-70^{\circ} \mathrm{N}$ zonal-mean zonal wind and polar cap temperature for the reduced NOGWD run and the increased NOGWD run. It is clear from the figure that the increased NOGWD run is unable to recreate the PJO event and instead produces two shallow and short-lived SSWs whose evolution is markedly different from the observations. The inability to recreate the PJO event in the increased NOGWD run results from the insufficient resolved wave forcing entering the stratosphere and markedly different basic state in the middle atmosphere.

Comparison of Figs. 7c-e and Figs. 7d-f reveals that reduction in NOGWD prolongs the persistence of the PJO event (from 24 to 38 days, quantified by the number of days the zonal-mean zonal wind is easterly at $60^{\circ} \mathrm{N}$ and $10 \mathrm{hPa}$ following the central date) and delays the reformation of the stratopause following the PJO event. This is made clearer by examining the difference in the zonalmean zonal wind and the polar cap temperature between the reduced NOGWD run and the control run in Figs. 9a,b. To better understand the response of the PJO life cycle to reduced NOGWD, the difference in the zonal wind tendencies and the residual vertical velocity between the reduced NOGWD run and the control run are shown in the left column of Fig. 8 (black and red contours).

Following the SSW, mesospheric westward NOGWD and the associated descent and adiabatic warming are suppressed in the reduced NOGWD run (Figs. 8a and 8g). Therefore, temperature evolves more diabatically in the mesosphere and the cooling is stronger in the reduced NOGWD run (blue shading in the mesosphere in Fig. 9b) as there is no wave drag to counteract the strong diabatic cooling. The much weaker descent following the warming in response to the reduction in NOGWD is in agreement with McLandress et al. (2013), who find a similar response in the run without any NOGWD. 
Because the PJO is more persistent in the reduced NOGWD run (i.e., the stratospheric zonal wind remains easterly for longer than in the control run), the ability of OGWs to propagate into the mesosphere is delayed in the reduced NOGWD run (Fig. 8c). This further contributes to the delay in the reformation of the stratopause. The stratopause begins to reform in the reduced NOGWD run only when sufficient parameterized and resolved wave drag is able to enter the mesosphere. The delay in the reformation of the stratopause was also observed by McLandress et al. (2013) in response to the removal of NOGWD.

The polar cap temperature in the lower stratosphere is colder in the reduced NOGWD run as the NOGWDinduced downwelling is suppressed. Therefore, the westerlies in the lower stratosphere are stronger in the reduced NOGWD run following the SSW. This allows more planetary waves to enter the stratosphere and induce resolved wave downwelling that contributes to the longer persistence of the PJO. This can be seen in Figs. 8e and 8g, where the lower-stratospheric resolved wave drag and downwelling strength are stronger in the reduced NOGWD run.

Because the 2006 PJO event is not captured in the increased NOGWD run, instead the evolution of the SSW that started on 15 December 2006 in the increased NOGWD run is compared to the PJO event in the control run. Figures $9 \mathrm{c}, \mathrm{d}$ shows the difference in the $50^{\circ}-70^{\circ} \mathrm{N}$ zonal-mean zonal wind and the polar cap temperature between the $2006 \mathrm{PJO}$ event in the control run and the 15 December $2006 \mathrm{SSW}$ event in the increased NOGWD run. The zonal wind tendencies and the residual vertical velocity for the increased NOGWD run are shown in the right column of Fig. 8 (shading) together with the difference in these quantities between the increased NOGWD run and the control run (black and red contours). The response of the SSW life cycle to an increase in NOGWD is almost opposite to that in the reduced NOGWD case just discussed. The main difference is that the SSW life cycle in the increased NOGWD run occurs lower down and that considerably less resolved wave drag is needed to initiate the SSW in the increased NOGWD run (cf. Figs. 8e,f) because of the weakened vortex brought about by the NOGWD increase. In addition, OGWD plays less of a role in the reformation of the stratopause as OGWD decreases to compensate for the increase in NOGWD (see Fig. 8d). As the recovery from the SSW event is shorter in the increased NOGWD run, the vortex reforms allowing more planetary wave activity to enter the stratosphere and initiate another SSW in February.

\section{b. SSWs in the free-running model}

Is the longer persistence of an SSW and a prolonged recovery of the stratopause following an SSW with reduction in NOGWD merely a feature of the $2006 \mathrm{PJO}$ case study, or does it occur more generally following all SSW events in the model? To address this, composites of all SSWs from the free-running control run, reduced NOGWD run, and increased NOGWD run are shown in Fig. 10 together with the ERA-Interim composites from 1979 to 2016. The composites are constructed as in McLandress and Shepherd (2009b). In the figure, $60^{\circ} \mathrm{N}$ zonal wind anomaly at $10 \mathrm{hPa}$, and the polar cap temperature anomaly at different pressure levels, are shown. It is clear from the figure that the response of the 2006 PJO event to the reduction in NOGWD carries over to SSWs in general. Namely, as the NOGWD is reduced, the persistence of the SSW events lengthens (i.e., the wind and temperature anomalies last longer), mainly because the amplitude of the events increase. Similarly, the reformation of the stratopause is delayed. An increase in NOGWD leads to opposite results. It should also be noted that the frequency of SSW events increases with increase in NOGWD: the frequency of SSWs is $0.45 \mathrm{yr}^{-1}$ for the reduced NOGWD run, $0.6 \mathrm{yr}^{-1}$ for the control run, and $0.9 \mathrm{yr}^{-1}$ for the increased NOGWD run. This is expected as a weaker vortex in the increased NOGWD run is more amenable to wave breaking. Note that the control run captures the statistical behavior of SSWs in the ERA-Interim remarkably well. The frequency of SSWs in the ERA-Interim reanalysis is $0.55 \mathrm{yr}^{-1}$.

\section{Summary and conclusions}

The impact of parameterized nonorographic gravity wave drag on key aspects of polar stratospheric dynamics was studied using the high-resolution IFS model. The focus was on the seasonal cycle of the residual mean meridional circulation, the SH vortex breakdown event, and NH SSWs.

Compared to the multimodel mean of Butchart et al. (2011), which was based on much lower-resolution models, the parameterized waves play a much smaller role in driving the tropical upwelling in the control IFS run (less than $7 \%$ everywhere in the stratosphere). The tropical upwelling is mostly influenced by resolved wave breaking in the lower stratosphere. However, the parameterized waves play a more important role in the winter polar cap downwelling, especially in the mid- to upper stratosphere and in particular over the $\mathrm{SH}$ winter pole. For example, at $10 \mathrm{hPa}$ parameterized waves account for $40 \%$ of the polar cap downwelling (all NOGWs) in the SH and $19 \%$ of the polar cap downwelling (14\% OGWs, $5 \%$ NOGWs) in the NH. Therefore, the residual mean meridional circulation is strongly influenced by NOGWD in the SH. 

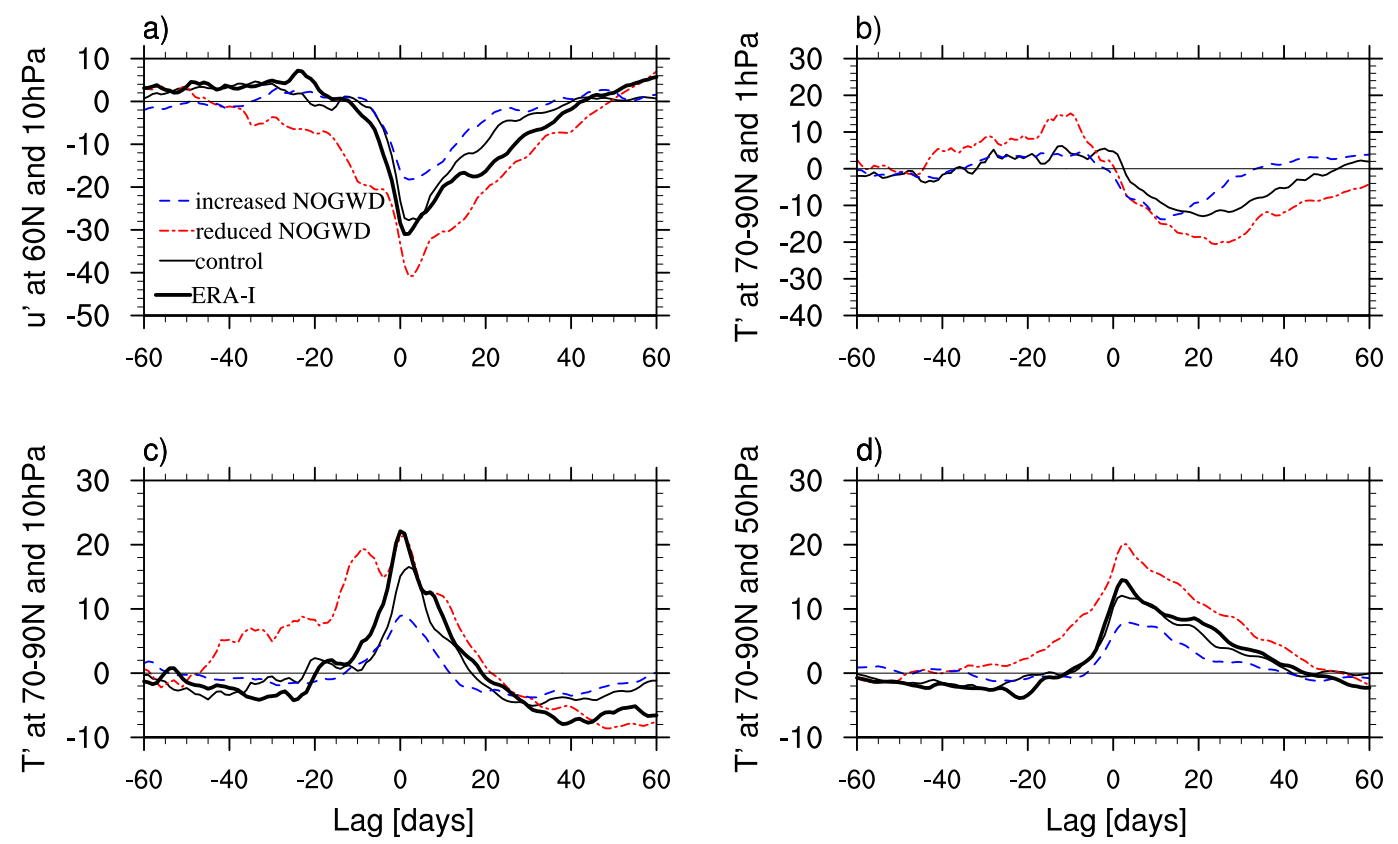

FIG. 10. Composites of all SSWs for the control run (solid black), reduced NOGWD run (dotted-dashed red), and increased NOGWD run (dashed blue) with the free-running model. Thick black line shows composites of SSWs from the ERA-Interim reanalysis between 1979 and 2016: (a) zonal-mean zonal wind anomaly at $60^{\circ} \mathrm{N}$ and $10 \mathrm{hPa}\left(\mathrm{m} \mathrm{s}^{-1}\right)$; and polar cap-averaged (from $70^{\circ}$ to $90^{\circ} \mathrm{N}$ ) zonal-mean temperature anomalies (K) at (b) $1 \mathrm{hPa}$, (c) $10 \mathrm{hPa}$, and (d) $50 \mathrm{hPa}$.

In response to changes in NOGWD flux, the resolved wave drag shifts vertically leading to a counteraction of the NOGWD perturbation in the polar mid- to upper stratosphere and an amplification of the perturbation in the polar lower stratosphere. Because of the different partitioning of the resolved and parameterized waves in driving the downwelling between the two hemispheres, the downwelling response in the total polar cap downwelling is different between the $\mathrm{NH}$ and the SH: the total downwelling increases with increase in NOGWD flux everywhere in the $\mathrm{SH}$, whereas in the $\mathrm{NH}$ it decreases in the mid- to upper stratosphere but increases in the lower stratosphere. OGWD also counteracts NOGWD changes in the NH.

The maximum in the parameterized and the resolved wave downwelling over the polar cap is found to have a temporal offset; the parameterized waves dominate earlier in the winter and the resolved waves dominate later in the winter/early spring. This offset is larger in the $\mathrm{SH}$. OGWs play no role in polar cap downwelling over the $\mathrm{SH}$ in the IFS. Because of the different seasonal cycles of the resolved and parameterized wave drags, the resolved and parameterized wave interaction does not occur on the Rossby wave propagation time scales when NOGWD is changed. During early winter, when the parameterized waves dominate the polar cap downwelling, the response is proportional to changes in
NOGWD. In the late winter/spring, however, the downwelling response is found to be dominated by the resolved waves. Therefore, the seasonal-mean perspective might paint a misleading picture of the resolved and parameterized wave interaction. In the $\mathrm{NH}$, the interaction with OGWD further complicates the matter. Therefore, it is unlikely that the NOGWD and OGWD parameterizations can be tuned independently, a conclusion also drawn in McLandress et al. (2013). It is, hence, easier to tune the NOGWD parameterization in the SH.

Despite having a much smaller influence on the timemean residual mean meridional circulation in the $\mathrm{NH}$, NOGWD has a clear effect on the SSW composites in the free-running model and on the $2006 \mathrm{PJO}$ event in the nudged model, in which the resolved wave fluxes entering the stratosphere are constrained to the observations. In particular, reduction in NOGWD leads to a reduction in the SSW frequency, increase in the amplitude and persistence, and a delay in the recovery of the stratopause following an SSW event. While the composites of SSW events in the control run agree well with ERA-Interim, this study illustrates that NOGWD flux exerts a strong influence on SSWs and might thus be a tunable parameter for obtaining a desired SSW behavior in other models. Moreover, the long-lived recovery period following SSWs represents a good opportunity to 
evaluate the accuracy of the model physics since the evolution is unaffected by chaotic variability.

Furthermore, the increase in NOGWD is found to bring forward the final warming date in the $\mathrm{SH}$ as the weakened vortex in the stratosphere is more amenable to wave breaking. Given that many stratosphereresolving chemistry-climate models have a late bias in the final warming date (Eyring et al. 2006; Butchart et al. 2011), it is possible that these models might be missing NOGWD. The final warming date in the control model climatology is, however, remarkably similar to the observed climatology and the IFS does not experience this late bias. Interestingly, Scheffler and Pulido (2015) find the opposite sign response in the final warming date in the stratosphere with changes to the NOGWD flux, with a delay in the final warming with an increase in NOGWD flux. This occurs because the planetary wave breaking in the lower stratosphere is reduced with increased NOGWD flux in their model, unlike in the IFS where the planetary wave forcing is markedly reduced in the upper stratosphere and mesosphere only.

As is shown here, the stratospheric circulation is profoundly influenced by NOGWD at TL255L137 resolution of the IFS, despite NOGWD mostly acting in the mesosphere and despite a greater role of the resolved gravity wave drag than in lower-resolution climate models. NOGWD exerts a strong influence on the polar night jet (in both hemispheres) and thus significantly alters the ability of resolved waves to influence stratospheric dynamics (i.e., the residual circulation, SSWs, and the final warming in the $\mathrm{SH}$ ). As the resolution of climate models increases, parameterized orographic gravity wave drag becomes less important in the middle atmosphere. Given that the strong sponge applied at the model top is likely to unphysically damp the smaller-scale higher-frequency inertia-gravity waves, nonorographic gravity wave drag parameterization will still be needed to substitute for this missing drag even in higher-resolution models with tops located in the mesosphere. Thus, NOGWD becomes the only parameterization affecting the momentum budget in the middle atmosphere at high resolution. Therefore, it is important to understand circulation sensitivity to NOGWD in order to guide interpretation and tuning of general circulation models.

Acknowledgments. This article is based on section 5 of the ECMWF technical memorandum of the authors (Polichtchouk et al. 2017). The authors thank Linus Magnusson and Tim Stockdale for help with setting up the IFS runs, Peter Hitchcock for providing gridded MLS temperature data, Nick Byrne for helpful discussions, and the three anonymous reviewers for their helpful comments. IP and TGS are supported by the
"Understanding the Atmospheric Circulation Response to Climate Change" (ACRCC, ERC Advanced Grant 339390) project.

\section{REFERENCES}

Andrews, D. G., J. R. Holton, and C. B. Leovy, 1987: Middle Atmosphere Dynamics. Academic Press, 489 pp.

Baldwin, M. P., and T. J. Dunkerton, 2001: Stratospheric harbingers of anomalous weather regimes. Science, 294, 581-584, https://doi.org/10.1126/science.1063315.

Black, R. X., and B. A. McDaniel, 2007: Interannual variability in the Southern Hemisphere circulation organized by stratospheric final warming events. J. Atmos. Sci., 64, 2968-2974, https://doi.org/10.1175/JAS3979.1.

Butchart, N., 2014: The Brewer-Dobson circulation. Rev. Geophys., 52, 157-184, https://doi.org/10.1002/2013RG000448.

_ , and Coauthors, 2011: Multimodel climate and variability of the stratosphere. J. Geophys. Res., 116, D05102, https:// doi.org/10.1029/2010JD014995.

Charney, J. G., and P. G. Drazin, 1961: Propagation of planetaryscale disturbances from the lower into the upper atmosphere. J. Geophys. Res., 66, 83-109, https://doi.org/10.1029/ JZ066i001p00083.

Cohen, N. Y., E. P. Gerber, and O. Bühler, 2013: Compensation between resolved and unresolved wave driving in the stratosphere: Implications for downward control. J. Atmos. Sci., 70, 3780-3798, https://doi.org/10.1175/JAS-D-12-0346.1.

,-- , and - 2014: What drives the Brewer-Dobson circulation? J. Atmos. Sci., 71, 3837-3855, https://doi.org/10.1175/ JAS-D-14-0021.1.

Dee, D., and Coauthors, 2011: The ERA-Interim reanalysis: Configuration and performance of the data assimilation system. Quart. J. Roy. Meteor. Soc., 137, 553-597, https://doi.org/ 10.1002/qj.828.

Douville, H., 2009: Stratospheric polar vortex influence on northern hemisphere winter climate variability. Geophys. Res. Lett., 36, L18703, https://doi.org/10.1029/2009GL039334.

ECMWF, 2016: IFS documentation. CY43R1, accessed 1 October 2017, http://www.ecmwf.int/en/forecasts/documentation-andsupport/changes-ecmwf-model/ifs-documentation.

Eyring, V., and Coauthors, 2006: Assessment of temperature, trace species, and ozone in chemistry-climate model simulations of the recent past. J. Geophys. Res., 111, D22308, https://doi.org/ 10.1029/2006JD007327.

Fritts, D. C., and M. J. Alexander, 2003: Gravity wave dynamics and effects in the middle atmosphere. Rev. Geophys., 41, 1003, https://doi.org/10.1029/2001RG000106.

Haynes, P. H., C. J. Marks, M. E. McIntyre, T. G. Shepherd, and K. P. Shine, 1991: On the "downward control" of extratropical diabatic circulations by eddy-induced mean zonal forces. J. Atmos. Sci., 48, 651-678, https://doi.org/10.1175/ 1520-0469(1991)048<0651:OTCOED > 2.0.CO;2.

Hitchcock, P., and T. G. Shepherd, 2013: Zonal-mean dynamics of extended recoveries from stratospheric sudden warmings. J. Atmos. Sci., 70, 688-707, https://doi.org/10.1175/JAS-D-120111.1.

— spheric sudden warmings. J. Atmos. Sci., 71, 3856-3876, https://doi.org/10.1175/JAS-D-14-0012.1.

_, T. G. Shepherd, and G. L. Manney, 2013: Statistical characterization of Arctic polar-night jet oscillation events. 
J. Climate, 26, 2096-2116, https://doi.org/10.1175/JCLI-D12-00202.1.

Holton, J., 1990: On the global exchange of mass between the stratosphere and troposphere. J. Atmos. Sci., 47, 392-395, https://doi.org/10.1175/1520-0469(1990)047<0392: OTGEOM $>2.0 . \mathrm{CO} ; 2$.

Kushner, P. J., and L. M. Polvani, 2004: Stratosphere-troposphere coupling in a relatively simple AGCM: The role of eddies. J. Climate, 17, 629-639, https://doi.org/10.1175/ 1520-0442(2004)017<0629:SCIARS $>2.0$. CO; 2 .

Limpasuvan, V., J. H. Richter, Y. J. Orsolini, F. Stordal, and O.-K. Kvissel, 2012: The roles of planetary and gravity waves during a major stratospheric sudden warming as characterized in WACCM. J. Atmos. Sol.-Terr. Phys., 78-79, 84-98, https:// doi.org/10.1016/j.jastp.2011.03.004.

Livesey, N. J., and Coauthors, 2011: Earth Observing System (EOS) Aura Microwave Limb Sounder MLS version 3.3 level 2 data quality and description document. Jet Propulsion Laboratory Rep. JPL D-33509, 156 pp., https://mls.jpl.nasa.gov/ data/v3-3_data_quality_document.pdf.

Lott, F., and M. J. Miller, 1997: A new subgrid-scale orographic drag parametrization: Its formulation and testing. Quart. J. Roy. Meteor. Soc., 123, 101-127, https://doi.org/10.1002/ qj. 49712353704.

McLandress, C., and T. G. Shepherd, 2009a: Simulated anthropogenic changes in the Brewer-Dobson circulation, including its extension to high latitudes. J. Climate, 22, 1516-1540, https:// doi.org/10.1175/2008JCLI2679.1.

$\longrightarrow$, and _ 2009b: Impact of climate change on stratospheric sudden warmings as simulated by the Canadian Middle Atmosphere Model. J. Climate, 22, 5449-5463, https://doi.org/ 10.1175/2009JCLI3069.1.

_, , S. Polavarapu, and S. R. Beagley, 2012: Is missing orographic gravity wave drag near $60^{\circ} \mathrm{S}$ the cause of the stratospheric zonal wind biases in chemistry-climate models? J. Atmos. Sci., 69, 802-818, https://doi.org/10.1175/JAS-D-110159.1.

_ J. F. Scinocca, T. G. Shepherd, M. C. Reader, and G. L. Manney, 2013: Dynamical control of the mesosphere by orographic and nonorographic gravity wave drag during the extended northern winters of 2006 and 2009. J. Atmos. Sci., 70, 2152-2169, https://doi.org/10.1175/JAS-D-12-0297.1.

Orr, A., P. Bechtold, J. Scinocca, M. Ern, and M. Janiskova, 2010: Improved middle atmosphere climate and forecasts in the ECMWF model through a nonorographic gravity wave drag parameterization. J. Climate, 23, 5905-5926, https://doi.org/ 10.1175/2010JCLI3490.1.

Plougonven, R., and F. Zhang, 2014: Internal gravity waves from atmospheric jets and fronts. Rev. Geophys., 52, 33-76, https:// doi.org/10.1002/2012RG000419.

Polavarapu, S., T. G. Shepherd, Y. Rochon, and S. Ren, 2005: Some challenges of middle atmosphere data assimilation. Quart. J. Roy. Meteor. Soc., 131, 3513-3527, https://doi.org/10.1256/ qj.05.87.

Polichtchouk, I., R. Hogan, T. Shepherd, P. Bechtold, T. Stockdale, S. Malardel, S.-J. Lock, and L. Magnusson, 2017: What controls the middle atmosphere circulation in the IFS? ECMWF Tech. Rep. 809, 48 pp., https://www.ecmwf.int/sites/default/ files/elibrary/2017/17670-what-influences-middle-atmospherecirculation-ifs.pdf.

Pulido, M., and J. Thuburn, 2008: The seasonal cycle of gravity wave drag in the middle atmosphere. J. Climate, 21, 46644679, https://doi.org/10.1175/2008JCLI2006.1.
Quintanar, A. I., and C. R. Mechoso, 1995: Quasi-stationary waves in the Southern Hemisphere. Part I: Observational data. J. Climate, 8, 2659-2672, https://doi.org/10.1175/15200442(1995)008<2659:QSWITS>2.0.CO;2.

Randel, W. J., 1988: The seasonal evolution of planetary waves in the southern hemisphere stratosphere and troposphere. Quart. J. Roy. Meteor. Soc., 114, 1385-1409, https://doi.org/ 10.1002/qj.49711448403.

_- R. Garcia, and F. Wu, 2008: Dynamical balances and tropical stratospheric upwelling. J. Atmos. Sci., 65, 3584-3595, https:// doi.org/10.1175/2008JAS2756.1.

Ren, S., S. M. Polavarapu, and T. G. Shepherd, 2008: Vertical propagation of information in a middle atmosphere data assimilation system by gravity-wave drag feedbacks. Geophys. Res. Lett., 35, L06804, https://doi.org/10.1029/ 2007GL032699.

Sakazaki, T., T. Sasaki, M. Shiotani, Y. Tomikawa, and D. Kinnison, 2015: Zonally uniform tidal oscillations in the tropical stratosphere. Geophys. Res. Lett., 42, 9553-9560, https://doi.org/10.1002/2015GL066054.

Scheffler, G., and M. Pulido, 2015: Compensation between resolved and unresolved wave drag in the stratospheric final warmings of the Southern Hemisphere. J. Atmos. Sci., 72, 4393-4411, https://doi.org/10.1175/JAS-D-14-0270.1.

, and _ 2017: Estimation of gravity-wave parameters to alleviate the delay in the Antarctic vortex breakup in general circulation models. Quart. J. Roy. Meteor. Soc., 143, 21572167, https://doi.org/10.1002/qj.3074.

Scinocca, J. F., 2003: An accurate spectral nonorographic gravity wave drag parameterization for general circulation models. J. Atmos. Sci., 60, 667-682, https://doi.org/10.1175/15200469(2003)060<0667:AASNGW>2.0.CO;2.

Seviour, W. J., N. Butchart, and S. C. Hardiman, 2012: The Brewer-Dobson circulation inferred from ERA-Interim. Quart. J. Roy. Meteor. Soc., 138, 878-888, https://doi.org/ 10.1002/qj.966.

Shaw, T. A., M. Sigmond, T. G. Shepherd, and J. F. Scinocca, 2009: Sensitivity of simulated climate to conservation of momentum in gravity wave drag parameterization. J. Climate, 22, 27262742, https://doi.org/10.1175/2009JCLI2688.1.

Shepherd, T. G., 2000: The middle atmosphere. J. Atmos. Sol.-Terr. Phys., 62, 1587-1601, https://doi.org/10.1016/ S1364-6826(00)00114-0.

Sigmond, M., and T. G. Shepherd, 2014: Compensation between resolved wave driving and parameterized orographic gravity wave driving of the Brewer-Dobson circulation and its response to climate change. J. Climate, 27, 5601-5610, https:// doi.org/10.1175/JCLI-D-13-00644.1.

, J. F. Scinocca, V. V. Kharin, and T. G. Shepherd, 2013: Enhanced seasonal forecast skill following stratospheric sudden warmings. Nat. Geosci., 6, 98-102, https://doi.org/10.1038/ ngeo1698.

Siskind, D. E., S. D. Eckermann, J. P. McCormack, L. Coy, K. W. Hoppel, and N. L. Baker, 2010: Case studies of the mesospheric response to recent minor, major, and extended stratospheric warmings. J. Geophys. Res., 115, D00N03, https://doi.org/10.1029/2010JD014114.

Tomikawa, Y., K. Sato, S. Watanabe, Y. Kawatani, K. Miyazaki, and M. Takahashi, 2012: Growth of planetary waves and the formation of an elevated stratopause after a major stratospheric sudden warming in a T213L256 GCM. J. Geophys. Res., 117, D16101, https://doi.org/10.1029/2011JD017243. 